\title{
The Effectiveness of Psychosocial Interventions with Cancer Patients: An Integrative Review of the Literature (2006-2011)
}

\author{
Bonnie Raingruber \\ California State University, Sacramento and University of California, Davis, Sacramento, CA 95817, USA \\ Correspondence should be addressed to Bonnie Raingruber, bonnie.raingruber@ucdmc.ucdavis.edu \\ Received 14 June 2011; Accepted 4 August 2011 \\ Academic Editors: A. Green and H. S. Shin
}

Copyright () 2011 Bonnie Raingruber. This is an open access article distributed under the Creative Commons Attribution License, which permits unrestricted use, distribution, and reproduction in any medium, provided the original work is properly cited.

Background. Previous integrative literature reviews and meta-analyses have yielded conflicting results regarding the effectiveness of psychosocial interventions for cancer patients. Methods. An integrative review of the literature focused on 19 randomized, controlled trials (2006-2011) was completed to examine the effectiveness of psychosocial interventions for cancer patients. Eligibility criteria: Inclusion criteria were the study was an English language randomized controlled clinical trial. Results. Seven studies involved nurses. Eleven studies resulted in positive outcomes. Overall, study quality was limited. In eight studies the intervention was not adequately described, 7 studies did not contain a hypothesis, 4 did not include clear eligibility criteria, 10 studies did not randomize appropriately, 9 did not list recruitment dates, 11 did not include a power analysis, 14 did not include blinded patients or data collectors, 11 did not use an intent-to-treat analysis, 10 did not clarify reasons for drop outs, and 11 did not discuss treatment fidelity. Conclusions. Future studies should build on previous findings, use comparable outcome measures, and adhere to standards of quality research. Qualitative studies are needed to determine what cancer patients of varied ages, cancer stages, and racial/ethnic backgrounds believe would be an effective intervention to manage their psychosocial needs.

\section{Introduction}

Therapeutic communication and supportive therapies are mainstays of mental health nursing practice. These psychosocial interventions are valuable adjuncts to physical treatment for individuals who have been diagnosed with cancer. It has been determined that $33 \%$ of individuals diagnosed with cancer experience severe psychological distress and up to $70 \%$ exhibit some degree of anxiety and depression [1-3]. Relationships, work life, and sense of self are all impacted by a cancer diagnosis [4].

Excellent care must include interventions that focus on the informational and psychosocial needs of patients [5]. Facilitating emotional expression helps to modulate distress and enhance coping abilities [6]. Psychosocial interventions including therapeutic communication have been used with success to minimize stress, improve quality of life, treat depression, and support cancer patients throughout the course of their diagnosis and recovery $[1,7]$.
A national symposium organized by the Agency for Health Care Research and Quality, the National Cancer Institute, and experts in the field concluded that communication and interventions targeting psychosocial issues were among the 8 key domains that are of vital importance in cancer care. These domains highlight the challenges that cancer patients face in coping with their emotions and navigating life disruptions associated with their treatment [8].

\section{Previous Meta-Analyses and Integrative Reviews}

A number of meta-analysis and reviews of the literature have focused on the effectiveness of psychosocial interventions with cancer patients. A meta-analysis conducted by Meyer and Mark [9] concluded that relaxation and behavioral modification improved functional adaptation and symptom control but did not affect medical outcomes. Newell et al. [10] reported that group-based and individual therapy, educational interventions, and guided imagery were effective 
with cancer patients. Most of these interventions were provided by a psychiatrist or psychologist, not nurses, and involved at least six contact hours. Barsevick et al. [11] conducted a systematic review of 36 studies to conclude psychoeducational interventions as well as behavior therapy reduced depression in cancer patients.

Rehse and Pukrop [12] conducted a meta-analysis of 37 controlled studies and found that psychosocial interventions improve quality of life among cancer patients. Studies between 1970 and 1999 published in English and German were included. Most studies were conducted in university settings and included primarily well-educated white participants. Females were twice as likely to be included given the frequency of breast cancer diagnoses. Only one outcome point was considered in the meta-analysis. An overall moderate effect sized of 0.31 was reported (even when Rosenthal's fail safe method was used to estimate unpublished studies). The duration of the intervention (greater than 15 weeks) was the only significant predictor that remained after controlling for other variables. The authors suggested that educational programs were more effective because other psychosocial interventions consisted of heterogeneous techniques while education was more consistently defined and implemented across studies.

Uitterhoeve et al. [13] reviewed the literature to examine the effectiveness of psychosocial interventions (specifically cognitive behavioral therapy) on quality of life for individuals diagnosed with advanced cancer. Thirteen studies published between 1990 and 2002 were critiqued. Nurses delivered the majority of interventions ( $n=8$ studies). In 12 of the 13 trials positive quality of life changes including improved levels of depression were seen.

Also in 2004, Chow et al. [14] conducted a meta-analysis on randomized controlled trials published between 1966 and 2002 and reported that psychosocial intervention did not prolong survival among cancer patients. Only 8 trials were reviewed, patients with differing types of metastatic illness were included, and trials with short follow-up times were reviewed, all of which limited the conclusiveness of the results.

Williams and Dale [15] published a systematic literature review that questioned whether psychotherapeutic interventions are effective in reducing depression among cancer patients. Their review included 18 trials published between 1995 and 2005. All studies provided clear descriptions of the intervention and had representative samples. Sixteen of the studies reported reasons patients gave for dropping out of the study. Several trials found cognitive behavioral therapy and social support reduced symptoms of depression. The major flaw identified by the author was that numerous studies were single-centre trials that did not monitor for use of other interventions that could have confounded the results.

Also in 2006 Osborn et al. [16] published a metaanalysis of the effectiveness of psychosocial interventions for depression, anxiety, and quality of life among cancer survivors. Included were 15 randomized controlled trials published between 1993 and 2004. Cognitive behavioral therapy was described as being effective in reducing depression, minimizing anxiety, and improving quality of life. Individual interventions were more effective than group interventions.

Jacobsen and Jim [17] summarized the results of systematic reviews and meta-analysis of the effects of psychosocial intervention on anxiety and depression among cancer patients. Randomized controlled trials between 1980 and 2003 were examined. Fourteen publications made reference to anxiety and 6 reported positive outcomes from psychosocial interventions. Nine studies reported psychosocial interventions were effective in improving symptoms of depression. The authors critiqued numerous methodological issues in the studies that were reviewed.

Edwards and Hulber-Williams [18] published a review of psychological interventions for women with metastatic breast cancer on psychological and survival outcomes. Randomized clinical trials published between 1966 and 2006 were examined. Five studies (cognitive behavioral and supportive-expressive) used a group format and showed limited evidence of benefit. Edwards and Hulber-Williams argued for standardization of outcome instruments, inclusion of cost effectiveness analysis and suggested interventions be designed based on preferences of individuals with cancer.

This integrative review of the literature was conducted to examine whether recent research has shown psychosocial interventions to be effective with cancer patients. The review summarizes randomized controlled studies and integrative reviews that have been published between 2006 and 2010 . During that period strides were made in medical treatment and knowledge of the influence of cortisol levels [19]. Patient attitudes toward psychosocial intervention may have shifted, reimbursement policies have been modified, and types of practitioners involved in provision of care have changed. Insufficient guidance currently exists for health care providers and researchers regarding the most effective type of psychosocial care [20]. It is necessary to understand whether, how, and why psychosocial interventions are effective with individuals who have been diagnosed with cancer. It is also critical to examine published studies if we are to design additional research that builds in a progressive manner on existing studies. If gaps in the literature are narrowed by successive refinements of psychosocial interventions and research methods we will have a more coherent research base from which to design future clinical interventions and research.

\section{Methods}

An integrative review of the literature, not a meta-analysis, designed to determine effect size was conducted. Electronic searches of Pub Med, Cinahl, and the Cochrane Library between 1/1/2006 (since the last published integrative literature review and meta-analysis) and 2/23/2011 were completed using the key words listed in Table 1. The type of research design was not specified in this search. This was done to identify as many relevant articles as possible and to avoid use of the search terms "randomized controlled trial" that might have resulted in missing relevant articles. Patients with cancer of any type or stage were included, and 
TABLE 1: Search terms used.

\begin{tabular}{lc}
\hline Keywords & Total articles located \\
\hline Therapeutic communication and cancer & 96 \\
Psychological support and cancer & 375 \\
Psychosocial and cancer & 174 \\
Communication and cancer & 308 \\
\hline
\end{tabular}

the review was not limited to a specific outcome measure such as depression or quality of life. This was done because given the small number of studies located $(n=19)$ it was not possible to target specific outcome measures such as depression or quality of life although several studies documented decreased depression and decreased anxiety $[7,21-23]$, improved quality of life $[23,24]$, and enhanced functional status/well-being $[25,26]$. References of all 19 papers were examined using the snowball method to identify additional studies. No studies that met the inclusion criteria were identified using the snowball method. No unpublished studies were located.

Of the above 953 articles initially identified, 42 were located with more than one search term and eliminated for being duplicative. Eight hundred forty-four studies were eliminated for not being randomized controlled trials. Only 27 (19 included +8 excluded) studies identified using search terms were randomized controlled trials. Inclusion criteria were that the study was a randomized controlled clinical trial published in English, involving adult cancer patients living at home who were over the age of $18(n=19)$. Randomized controlled trials that used volunteers to provide the intervention, focused on cancer patients not living at home, included patient spouses in the intervention, or included pharmacological, physical therapy, and/or complementary and alternative interventions were excluded.

Eight randomized controlled trials were eliminated for not meeting inclusion criteria. One study was excluded as it was a composite of hypnosis (an alternative treatment) and supportive-expressive group therapy [27]. One study was eliminated because it included an intervention that included conditioning exercises provided by a physical therapist [28]. Another study was eliminated because it included a couplefocused group intervention [29]. Two studies were excluded because they combined psychosocial interventions along with use of medications in studies involving cancer patients $[1,30]$. One study was excluded because it focused on elderly individuals with cancer living in care homes rather than at home [21]. One study was eliminated because it used volunteers to provide the intervention [24]. One study was eliminated because it reported the qualitative results obtained from a randomized controlled trial [20]. A data extraction form which is labeled Table 2 was developed based on Consolidated Standards of Reporting Trials (-CONSORT) guidelines [22] and used to summarize the included studies and to rank their quality.

\section{Results}

Nineteen randomized controlled trials focusing on therapeutic communication and supportive interventions were grouped into four main categories: cognitive behavioral interventions, supportive interventions, group interventions, and telephone-assisted interventions. Of the 19 studies reviewed 11 studies demonstrated positive findings [7, 23, $25,26,31,32,34,35,38,40,41]$ while an additional 4 demonstrated positive findings only after a post hoc analysis was completed $[5,19,33,36]$. Findings from each category of article are described below.

4.1. Cognitive Behavioral Interventions. Cognitive behavioral interventions were based on the belief that how individuals view situations influences their emotional response and problem-solving ability. Cognitive behavioral interventions are summarized in Table 3. Antoni et al. [7, 32] reported different outcome measures from a single study of female breast cancer patients. In one publication the intervention group had lower anxiety and cortisol and higher cytokine production than the control group [32]. In the second article, a greater reduction in cancer-specific thought intrusion, anxiety, and emotional distress was described in the cognitive behavioral intervention group [7]. Miller et al. [25] reported quality of life improved for patients with colorectal, head/neck, lung, and breast cancer who participated in 8 sessions of a cognitive behavioral intervention. Pitceathly et al. [26] found high-risk patients with breast, lymphoma, or gynecological cancer who received cognitive behavioral therapies were less likely to become depressed or anxious.

4.2. Supportive Interventions. Randomized controlled trials focused on supportive psychosocial interventions that included listening, validation, stress management, problem solving, and education related to the diagnosis. Interventions in this category varied substantially including (1) a onetime meeting with a psychologist for clients with gynecological cancer [41], (2) two communication skills training sessions with nurses for clients with gastric, colorectal, or breast cancer [40], (3) a coping and communicationenhancing intervention, supportive counseling or usual care intervention offered in six hour-long sessions with a therapist for patients with gynecological cancers [35], and (4) an educational and stress management intervention lasting 2 hours for patients with melanoma [39]. A coping and communication support intervention provided by mental health nurses who offered an in-home meeting and a followup telephone contact for patients with lung, pancreatic, or liver cancer [42] is discussed in the telephone intervention section even though it is both supportive and telephone based. Supportive interventions are summarized in Table 4 .

Although each of the supportive interventions resulted in improved outcomes, each study used a different type of supportive care, was implemented by varied types of practitioners, relied on a spectrum of different outcome measures, and included clients with several types and stages of cancer. The use of varied interventions as well as the limitations listed in Table 2 made it difficult to make any definitive conclusions about these randomized, controlled trials. 
TABLe 2: Study quality.

\begin{tabular}{|c|c|c|}
\hline Quality measure & Author & Type of study \\
\hline A hypothesis is provided ( 1 point) & $\begin{array}{l}\text { Andersen et al. [31], Andersen et al. [23], Antoni et al. [7], Antoni et } \\
\text { al. [32], Aranda et al. [5], Girgis et al. [33], Kravitz et al. [34], Manne } \\
\text { et al. [35], Miller et al. [25], Pitceathy et al. [26], Spiegel et al. [36], } \\
\text { Walker et al. [37]. }\end{array}$ & $\begin{array}{l}4 \text { Cognitive behavioral } \\
1 \text { Supportive } \\
3 \text { Group } \\
4 \text { Telephone }\end{array}$ \\
\hline A hypothesis is not provided & $\begin{array}{l}\text { Andersen et al. [38], Boesen et al. [39], Fukui et al. [40], Kissane et al. } \\
\text { [19], Powell et al. [41] Rose et al. [6], Rose et al. [42]. }\end{array}$ & $\begin{array}{l}3 \text { Supportive } \\
2 \text { Group } \\
2 \text { Telephone }\end{array}$ \\
\hline Eligibility criteria are clear (1 point) & $\begin{array}{l}\text { Andersen et al. [31], Andersen et al. [38], Andersen et al. [23], Antoni } \\
\text { et al. [7], Antoni et al. [32], Boesen et al. [39], Fukui et al. [40], Girgis } \\
\text { et al. [33], Kissane et al. [19], Kravitz et al. [34], Manne et al. [35], } \\
\text { Rose et al. [6], Rose et al. [42], Spiegel et al. [36], Walker et al. [37]. }\end{array}$ & $\begin{array}{l}2 \text { Cognitive behavioral } \\
3 \text { Supportive } \\
5 \text { Group } \\
5 \text { Telephone }\end{array}$ \\
\hline Eligibility criteria are not clear & $\begin{array}{l}\text { Aranda et al. [5], Miller et al. [25], Pitceathy et al. [26], Powell et al. } \\
\text { [41]. }\end{array}$ & $\begin{array}{l}2 \text { Cognitive behavioral } \\
1 \text { Supportive } \\
1 \text { Telephone }\end{array}$ \\
\hline $\begin{array}{l}\text { The intervention is described with } \\
\text { sufficient detail it could be replicated } \\
\text { (1 point) }\end{array}$ & $\begin{array}{l}\text { Andersen et al. [31], Andersen et al. [38], Andersen et al. [23], Antoni } \\
\text { et al. [7], Antoni et al. [32], Aranda et al. [5], Kissane et al. [19], } \\
\text { Kravitz et al. [34], Pitceathy et al. [26], Rose et al. [6], Walker et al. } \\
\text { [37]. }\end{array}$ & $\begin{array}{l}3 \text { Cognitive behavioral } \\
4 \text { Group } \\
4 \text { Telephone }\end{array}$ \\
\hline $\begin{array}{l}\text { The intervention is not described with } \\
\text { sufficient detail to allow for replication }\end{array}$ & $\begin{array}{l}\text { Boesen et al. [39], Fukui et al. [40], Girgis et al. [33], Manne et al. } \\
\text { [35], Miller et al. [25], Powell et al. [41], Rose et al. [42], Spiegel et al. } \\
\text { [36]. }\end{array}$ & $\begin{array}{l}1 \text { Cognitive behavioral } \\
4 \text { Supportive } \\
1 \text { Group } \\
2 \text { Telephone }\end{array}$ \\
\hline $\begin{array}{l}\text { Treatment fidelity is discussed ( } 1 \\
\text { point) }\end{array}$ & $\begin{array}{l}\text { Andersen et al. [38], Antoni et al. [32], Fukui et al. [40], Kissane et al. } \\
\text { [19], Kravitz et al. [34], Pitceathy, et al. [26], Spiegel et al. [36], } \\
\text { Walker et al. [37]. }\end{array}$ & $\begin{array}{l}2 \text { Cognitive behavioral } \\
1 \text { Supportive } \\
3 \text { Group } \\
2 \text { Telephone }\end{array}$ \\
\hline Treatment fidelity is not addressed & $\begin{array}{l}\text { Andersen et al. [31], Andersen et al. [23], Antoni et al. [7], Aranda et } \\
\text { al. [5], Boesen et al. [39], Girgis et al. [33], Manne et al. [35], Miller et } \\
\text { al. [25], Powell et al. [41], Rose et al. [6], Rose et al. [42]. }\end{array}$ & $\begin{array}{l}2 \text { Cognitive behavioral } \\
3 \text { Supportive } \\
2 \text { Group } \\
4 \text { Telephone }\end{array}$ \\
\hline $\begin{array}{l}\text { A power analysis was provided to } \\
\text { establish the sample size ( } 1 \text { point })\end{array}$ & $\begin{array}{l}\text { Andersen et al. [23], Girgis et al. [33], Manne et al. [35], Miller et al. } \\
\text { [25], Pitceathy et al. [26], Powell et al. [41], Spiegel et al. [36], Walker } \\
\text { et al. [37]. }\end{array}$ & $\begin{array}{l}2 \text { Cognitive behavioral } \\
2 \text { Supportive } \\
2 \text { Group } \\
2 \text { Telephone }\end{array}$ \\
\hline No power analysis was included & $\begin{array}{l}\text { Andersen et al. [31], Andersen et al. [38], Antoni et al. [7], Antoni et } \\
\text { al. [32], Aranda et al. [5], Boesen et al. [39], Fukui et al. [40], Kissane } \\
\text { et al. [19], Kravitz et al. [34], Rose et al. [6], Rose et al. [42]. }\end{array}$ & $\begin{array}{l}2 \text { Cognitive behavioral } \\
2 \text { Supportive } \\
3 \text { Group } \\
4 \text { Telephone }\end{array}$ \\
\hline $\begin{array}{l}\text { The method used for randomization } \\
\text { and who randomized are described ( } 1 \\
\text { point) }\end{array}$ & $\begin{array}{l}\text { Andersen et al. [23], Aranda et al. [5], Girgis et al. [33], Kissane et al. } \\
\text { [19], Kravitz et al. [34], Miller et al. [25], Manne et al. [35], Pitceathy } \\
\text { et al. [26], Walker et al. [37]. }\end{array}$ & $\begin{array}{l}2 \text { Cognitive behavioral } \\
1 \text { Supportive } \\
2 \text { Group } \\
4 \text { Telephone }\end{array}$ \\
\hline $\begin{array}{l}\text { It is not clear how randomization was } \\
\text { done (method and responsible } \\
\text { individual) }\end{array}$ & $\begin{array}{l}\text { Andersen et al. [31], Andersen et al. [38], Antoni et al. [7], Antoni et } \\
\text { al. [32], Boesen et al. [39], Fukui et al. [40], Powell et al. [41], Rose et } \\
\text { al. [6], Rose et al. [42], Spiegel et al. [36]. }\end{array}$ & $\begin{array}{l}2 \text { Cognitive behavioral } \\
3 \text { Supportive } \\
3 \text { Group } \\
2 \text { Telephone }\end{array}$ \\
\hline Blinding was discussed (1 point) & $\begin{array}{l}\text { Andersen et al. [31], Fukui et al. [40], Kravitz et al. [34], Pitceathy et } \\
\text { al. [26], Walker et al. [37]. }\end{array}$ & $\begin{array}{l}1 \text { Cognitive behavioral } \\
1 \text { Supportive } \\
1 \text { Group } \\
2 \text { Telephone }\end{array}$ \\
\hline Blinding was not discussed & $\begin{array}{l}\text { Andersen et al. [38], Andersen et al. [23], Antoni et al. [7], Antoni et } \\
\text { al. [32], Aranda et al. [5], Boesen et al. [39], Girgis et al. [33], Kissane } \\
\text { et al. [19], Manne et al. [35], Miller et al. [25], Powell et al. [41], Rose } \\
\text { et al. [6], Rose et al. [42], Spiegel et al. [36]. }\end{array}$ & $\begin{array}{l}3 \text { Cognitive behavioral } \\
3 \text { Supportive } \\
4 \text { Group } \\
4 \text { Telephone }\end{array}$ \\
\hline
\end{tabular}


Table 2: Continued.

\begin{tabular}{|c|c|c|}
\hline Quality measure & Author & Type of study \\
\hline $\begin{array}{l}\text { Reasons for dropouts are given }(1 \\
\text { point) }\end{array}$ & $\begin{array}{l}\text { Antoni et al. [32], Boesen et al. [39], Fukui et al. [40], Girgis et al. } \\
\text { [33], Kissane et al. [19], Miller et al. [25], Pitceathy et al. [26], Powell } \\
\text { et al. [41], Spiegel et al. [36]. }\end{array}$ & $\begin{array}{l}3 \text { Cognitive behavioral } \\
3 \text { Supportive } \\
2 \text { Group } \\
1 \text { Telephone }\end{array}$ \\
\hline No reasons for dropouts are provided & $\begin{array}{l}\text { Andersen et al. [31], Andersen et al. [38], Andersen et al. [23], Antoni } \\
\text { et al. [7], Aranda et al. [5], Kravitz et al. [34], Manne et al. [35], Rose } \\
\text { et al. [6], Rose et al. [42], Walker et al. [37]. }\end{array}$ & $\begin{array}{l}1 \text { Cognitive behavioral } \\
3 \text { Group } \\
1 \text { Supportive } \\
5 \text { Telephone }\end{array}$ \\
\hline $\begin{array}{l}\text { An intent-to-treat analysis was } \\
\text { completed ( } 1 \text { point })\end{array}$ & $\begin{array}{l}\text { Andersen et al. [31], Andersen et al. [23], Antoni et al. [7], Kissane et } \\
\text { al. [19], Pitceathy et al. [26], Powell et al. [41], Spiegel et al. [36], } \\
\text { Walker et al. [37]. }\end{array}$ & $\begin{array}{l}2 \text { Cognitive behavioral } \\
1 \text { Supportive } \\
4 \text { Group } \\
1 \text { Telephone }\end{array}$ \\
\hline $\begin{array}{l}\text { An intent-to-treat analysis is not } \\
\text { mentioned }\end{array}$ & $\begin{array}{l}\text { Andersen et al. [38], Antoni et al. [32], Aranda et al. [5], Boesen et al. } \\
\text { [39], Fukui et al. [40], Girgis et al. [33], Kravitz et al. [34], Manne et } \\
\text { al. [35], Miller et al. [25], Rose et al. [6], Rose et al. [42]. }\end{array}$ & $\begin{array}{l}2 \text { Cognitive behavioral } \\
3 \text { Supportive } \\
1 \text { Group } \\
5 \text { Telephone }\end{array}$ \\
\hline $\begin{array}{l}\text { Recruitment dates are specified ( } 1 \\
\text { point) }\end{array}$ & $\begin{array}{l}\text { Andersen et al. [23], Boesen et al. [39], Fukui et al. [40], Kissane et al. } \\
\text { [19], Manne et al. [35], Miller et al. [25], Pitceathy et al. [26], Powell } \\
\text { et al. [41], Spiegel et al. [36], Walker et al. [37]. }\end{array}$ & $\begin{array}{l}2 \text { Cognitive behavioral } \\
4 \text { Supportive } \\
3 \text { Group } \\
1 \text { Telephone }\end{array}$ \\
\hline Recruitment dates are not specified & $\begin{array}{l}\text { Andersen et al. [31], Andersen et al. [38], Antoni et al. [7], Antoni et } \\
\text { al. [32], Aranda et al. [5], Girgis et al. [33], Kravitz et al. [34], Rose et } \\
\text { al. [6], Rose et al. [42]. }\end{array}$ & $\begin{array}{l}2 \text { Cognitive behavioral } \\
2 \text { Group } \\
5 \text { Telephone }\end{array}$ \\
\hline $\begin{array}{l}\text { Study limitations are described ( } 1 \\
\text { point) }\end{array}$ & $\begin{array}{l}\text { Andersen et al. [31], Andersen et al. [38], Antoni et al. [7], Antoni et } \\
\text { al. [32], Aranda et al. [5], Boesen et al. [39], Fukui et al. [40], Kissane } \\
\text { et al. [19], Girgis et al. [33], Kravitz et al. [34], Manne et al. [35], } \\
\text { Miller et al. [25], Pitceathy et al. [26], Powell et al. [41], Rose et al. } \\
\text { [6], Rose et al. [42], Spiegel et al. [36], Walker et al. [37]. }\end{array}$ & $\begin{array}{l}4 \text { Cognitive behavioral } \\
4 \text { Supportive } \\
4 \text { Group } \\
6 \text { Telephone }\end{array}$ \\
\hline $\begin{array}{l}\text { Study limitations are not described in } \\
\text { detail }\end{array}$ & Andersen et al. [23]. & 1 Group \\
\hline Significant results were reported & $\begin{array}{l}\text { Andersen et al. [31], Andersen et al. [38], Andersen et al. [23], Antoni } \\
\text { et al. [7], Antoni et al. [32], Aranda et al. [5], Fukui et al. [40], Girgis } \\
\text { et al. [33], Kissane et al. [19], Kravitz et al. [34], Manne et al. [35], } \\
\text { Miller et al. [25], Pitceathy et al. [26], Powell, et al. [41]. }\end{array}$ & $\begin{array}{l}4 \text { Cognitive behavioral } \\
3 \text { Supportive } \\
4 \text { Group } \\
3 \text { Telephone }\end{array}$ \\
\hline $\begin{array}{l}\text { Significant results were not reported. } \\
\text { Note: data collection continues for } \\
\text { Walker [37] and Rose [6]. }\end{array}$ & Boesen et al. [39], Rose et al. [42], Spiegel et al. [36]. & $\begin{array}{l}1 \text { Supportive } \\
1 \text { Group } \\
1 \text { Telephone }\end{array}$ \\
\hline Funding sources are listed (1 point) & $\begin{array}{l}\text { Andersen et al. [31], Andersen et al. [38], Andersen et al. [23], Antoni } \\
\text { et al. [7], Antoni et al. [32], Aranda et al. [5], Boesen et al. [39], Fukui } \\
\text { et al. [40], Kissane et al. [19], Girgis et al. [33], Kravitz et al. [34], } \\
\text { Manne et al. [35], Pitceathy et al. [26], Rose et al. [6], Rose et al. [42], } \\
\text { Spiegel et al. [36], Walker et al. [37]. }\end{array}$ & $\begin{array}{l}3 \text { Cognitive behavioral } \\
3 \text { Supportive } \\
5 \text { Group } \\
6 \text { Telephone }\end{array}$ \\
\hline A trial registry is mentioned ( 1 point $)$ & Girgis et al. [33], Kravitz et al. [34] & 2 Telephone \\
\hline \multirow{6}{*}{$\begin{array}{l}\text { Nurse-delivered treatment. Seven } \\
\text { studies included nurses in providing } \\
\text { the intervention. }\end{array}$} & $\begin{array}{l}\text { (i) Coled by a nurse, physical therapist, chaplain, or social worker } \\
\text { (Miller-CB, }[25] \text { ) }\end{array}$ & \\
\hline & (ii) One nurse, one social worker (Pitceathly-CB, [26]) & \\
\hline & (iii) 3 nurses provided the intervention (Fukui-S, [40]) & \\
\hline & (iv) Breast cancer nurses (Aranda-T, [5]) & \\
\hline & (v) Masters prepared psychiatric nurses (Rose-T, [6] and S/T, [42]) & \\
\hline & (vi) Cancer nurses (Walker-T, [37]) & \\
\hline
\end{tabular}


Table 2: Continued.

\begin{tabular}{|c|c|c|}
\hline Quality measure & Author & Type of study \\
\hline \multirow{16}{*}{$\begin{array}{l}\text { Length of study. The length of } \\
\text { interventions varied from a single visit } \\
\text { to one year. }\end{array}$} & Miller et al. (CB, [25]), 8 sessions/27 weeks & \\
\hline & Pitceathly et al. (CB, [26]), 3 sessions/6 weeks & \\
\hline & Antoni et al. (CB, $[7,32]), 2$ hour sessions/ 10 weeks & \\
\hline & Boesen et al. (S, [39]), 6, 2 hour sessions/6 weeks & \\
\hline & Fukui et al. (S, [40]), interviews at baseline, 1 month, 3 months & \\
\hline & Manne et al. (S, [35]), 6 sessions unclear timeframe & \\
\hline & Powell et al. (S, [41]), single visit & \\
\hline & $\begin{array}{l}\text { Andersen et al. (G, }[23,31,38]), 4 \text { months weekly followed by } 8 \\
\text { monthly for } 12 \text { months total }\end{array}$ & \\
\hline & Kissane et al. (G, [19]), 1 year & \\
\hline & Spiegel et al. (G, [36]), 1 year & \\
\hline & $\begin{array}{l}\text { Kravitz et al. (T, [34]), } 1 \text { session/telephone contact at 2, 6, and } 12 \\
\text { weeks. }\end{array}$ & \\
\hline & Aranda et al. (T, [5]), 1 session/1 telephone followup & \\
\hline & Rose et al. ( $\mathrm{T},[6]), 1$ session/6 weeks of telephone contact & \\
\hline & Rose et al. (S/T, [42]), 2 months & \\
\hline & Walker et al. (T, [37]), 8 sessions in 16 weeks and monthly telephone & \\
\hline & Girgis et al. (T, [33]), telephone contact every 6 weeks for 6 months & \\
\hline
\end{tabular}

4.3. Group Interventions. Several researchers conducted randomized controlled trials to explore the effectiveness of group approaches that included psychosocial interventions for individuals diagnosed with cancer. Group interventions are summarized in Table 5. As with the supportive approaches the actual group interventions that were used differed significantly. Interventions for individuals with breast cancer included (1) 26 group sessions offered over a oneyear period led by clinical psychologists $[23,31,38]$, (2) 1 year of group therapy offered by psychologists, psychiatrists, or social workers [19], and (3) weekly supportive expressive group therapy and education lead by psychiatrists, psychologists, and social workers [36]. The length and number of sessions offered, the professionals involved, and the outcome measures used in these studies varied.

One meta-analysis was also published on the effectiveness of cancer support groups. This meta-analysis covered the years of 1981 to 2001 and included 20 randomized controlled trials. Results indicated that support group participation results in decreased depression and anxiety, increased illness adaptation, improved quality of life, and enhanced marital relationships. Group interventions did not impact survival. Forty-five percent of principal investigators were psychologists, 35\% were nurses, and 20\% were physicians. Seventy $\%$ of studies involved women, $65 \%$ focused on women with breast cancer, $96 \%$ offered group sessions on a weekly basis, and a cognitive-behavioral model was used in $92 \%$ of studies. Twelve studies focused on depression, 11 on anxiety, 6 on quality of life, 8 on adaptation to illness, 3 on survival, and 2 on marital relationships [45].
4.4. Telephone-Assisted Interventions. Six randomized controlled trials examined support provided by telephone. These telephone assisted interventions are summarized in Table 6. Telephone-assisted interventions typically included in-person contacts to establish rapport, followed by periodic telephone contact and support [5, 6, 34, 37, 42]. Kravitz et al. [34] and Girgis et al. [33] compared telephone-based care to care provided by a physician. Two studies included family members in the initial visit $[5,6]$. In-person contacts among the telephone studies ranged from 1 session $[5,6]$ to 6 to 8 sessions [37]. In these studies challenges such as symptoms limiting travel, geography, and intervention-associated costs provided the rationale for using telephone-assisted support [20]. Telephone contact was used because it can be a flexible way to provide timely intervention during a stressful period [6].

The studies by Rose et al. $[6,42]$ were based on the same sample, collected at 6 weeks [6] showing middle-aged patients had more problems communicating with family members and at 2 months [42] showing middle-aged patients averaged more contacts per month compared to individuals aged 61 to 80 .

Aranda et al. [5] used a brief, nurse-delivered visit followed by one phone call. The intervention was not more effective than usual care. The authors postulated that the intervention was too brief to be effective. When the results were analyzed only with women with high needs the intervention was effective in reducing psychological and emotional needs. 


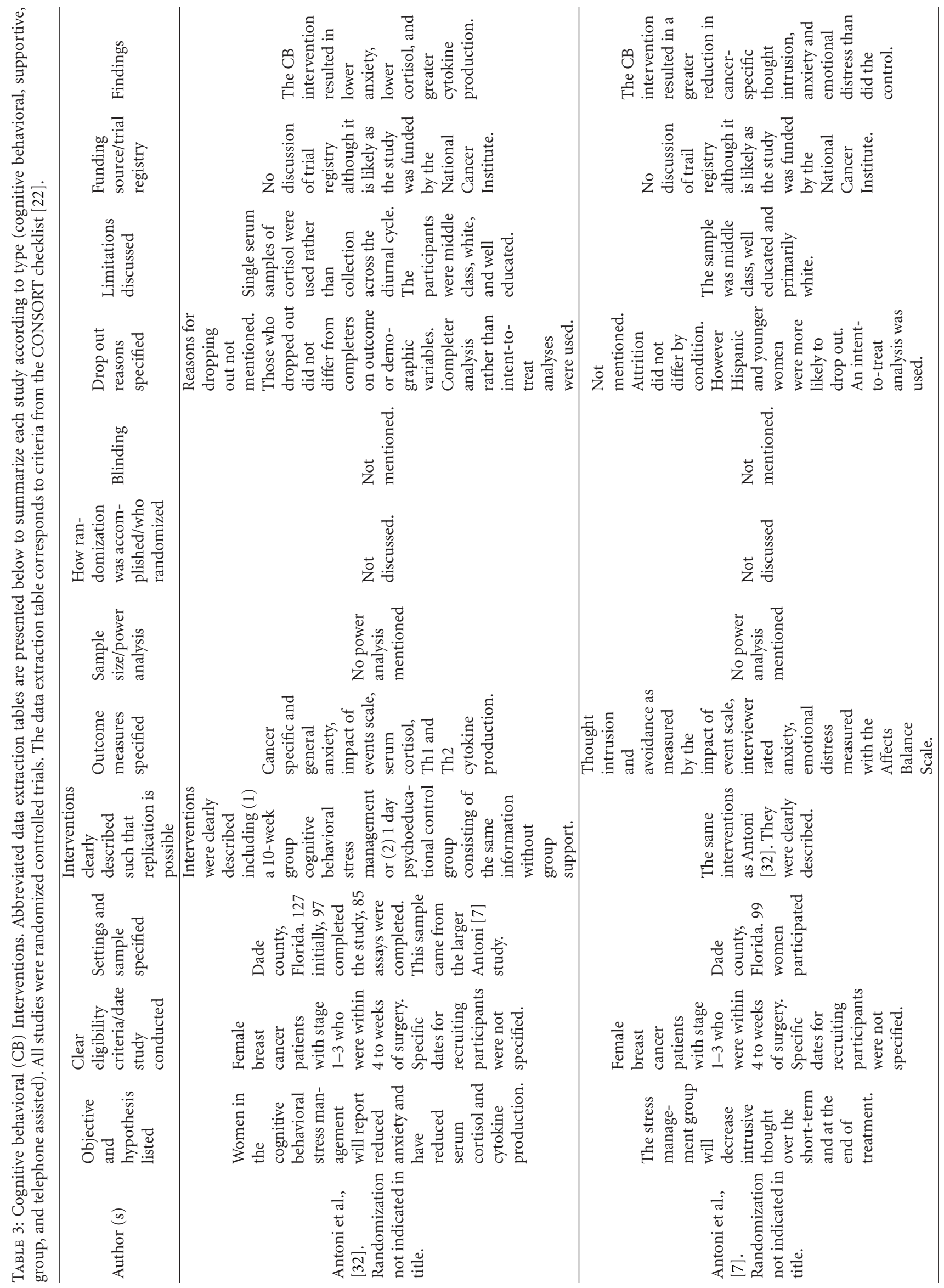




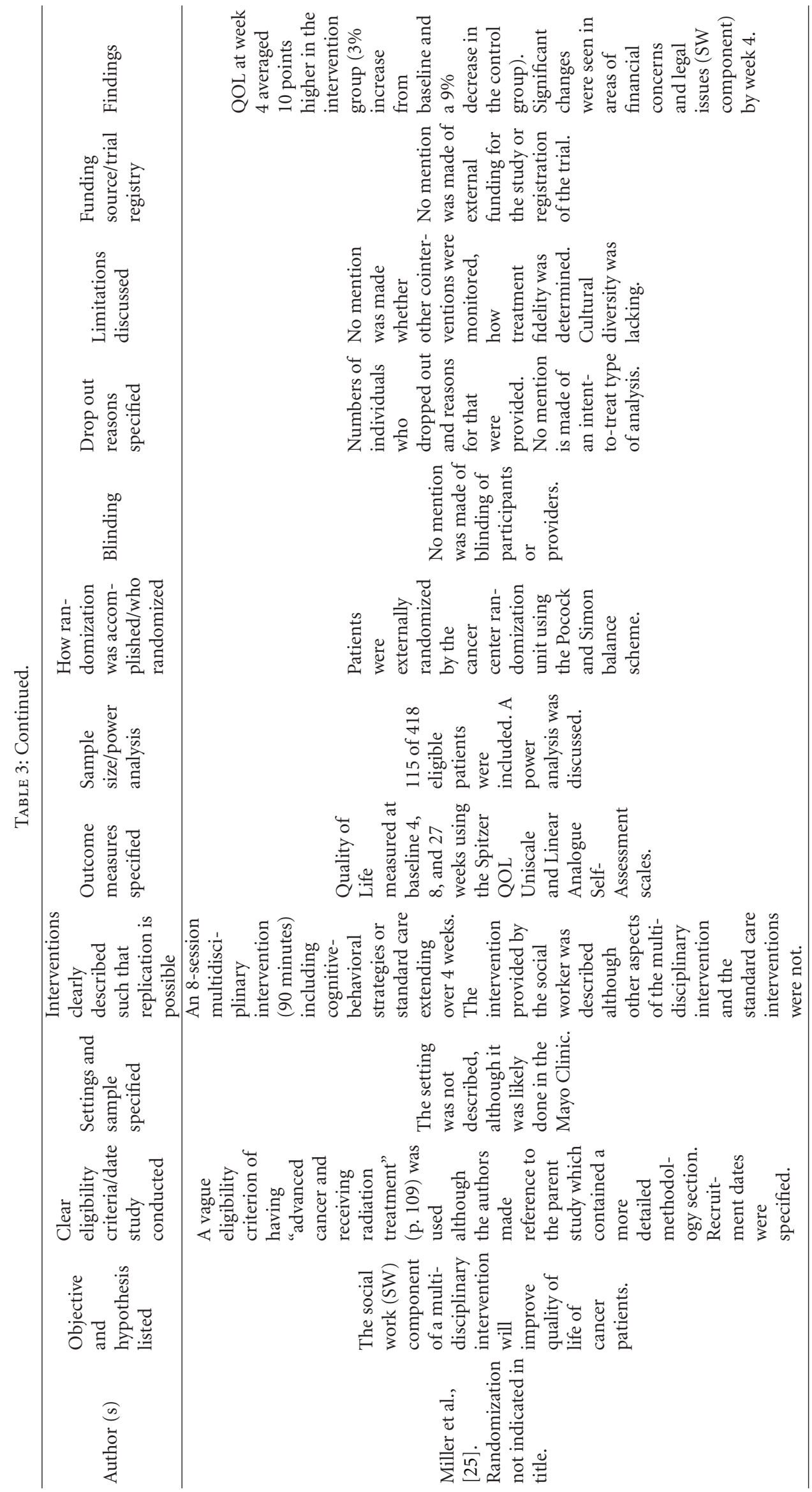




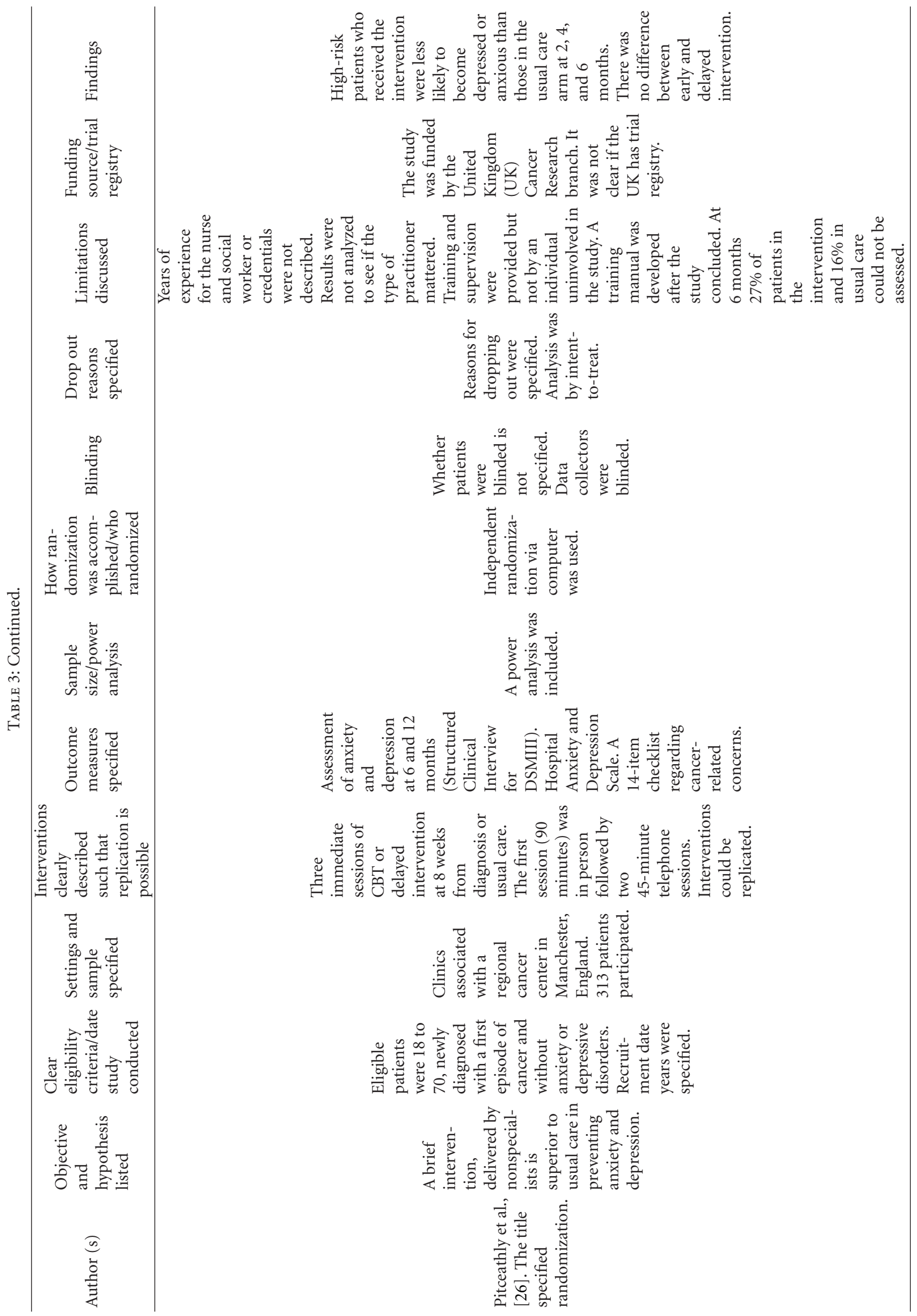




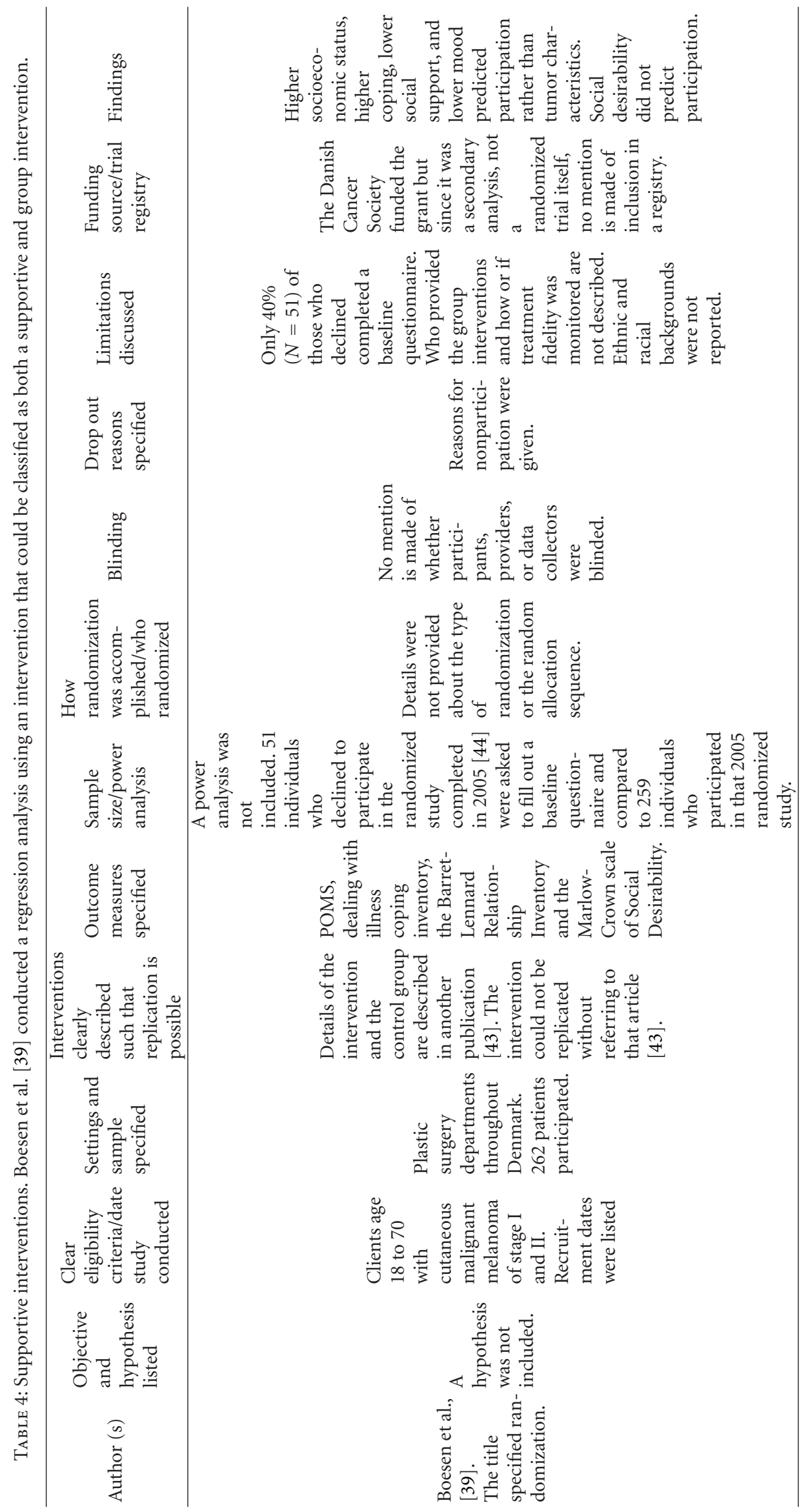




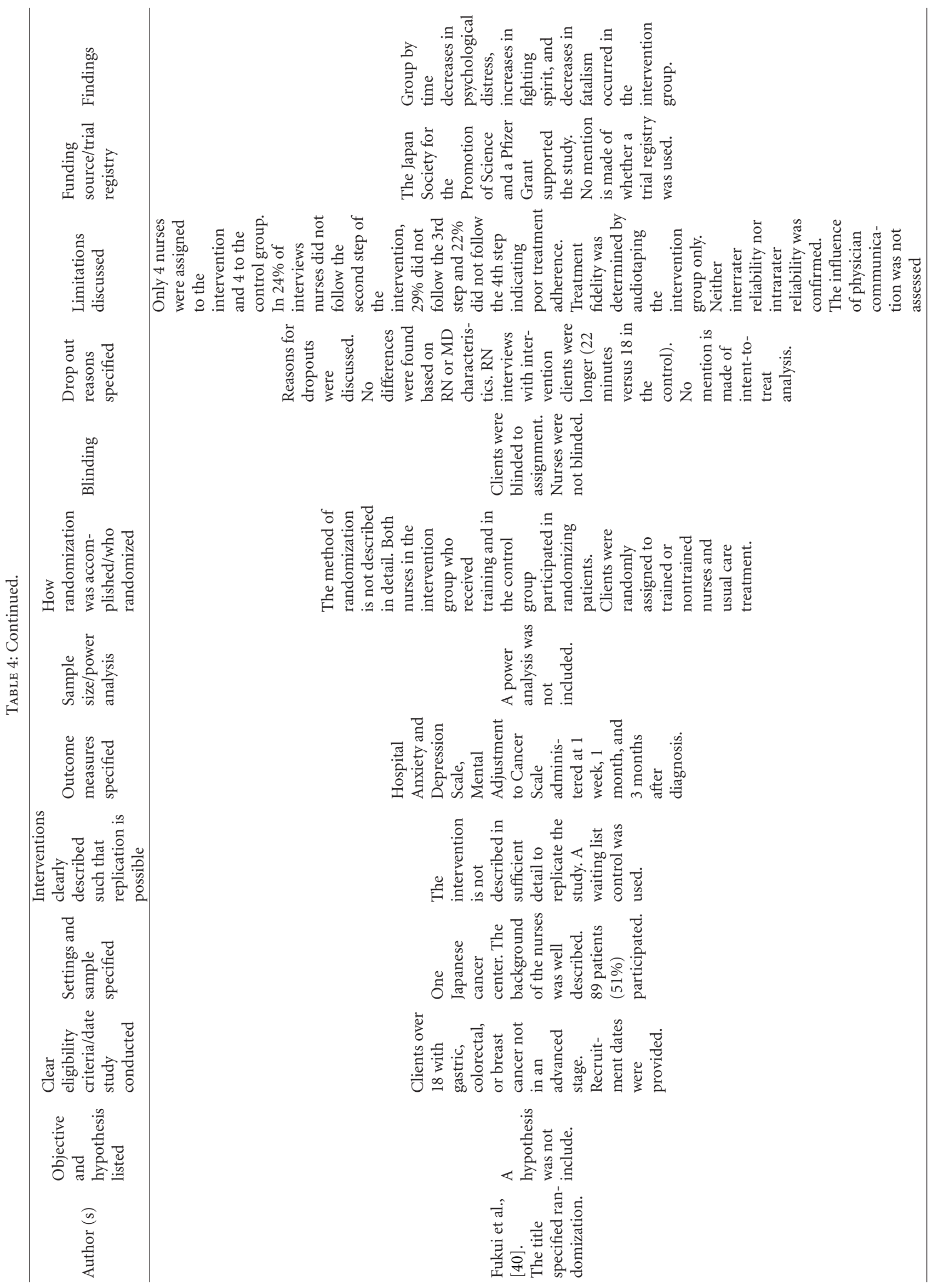




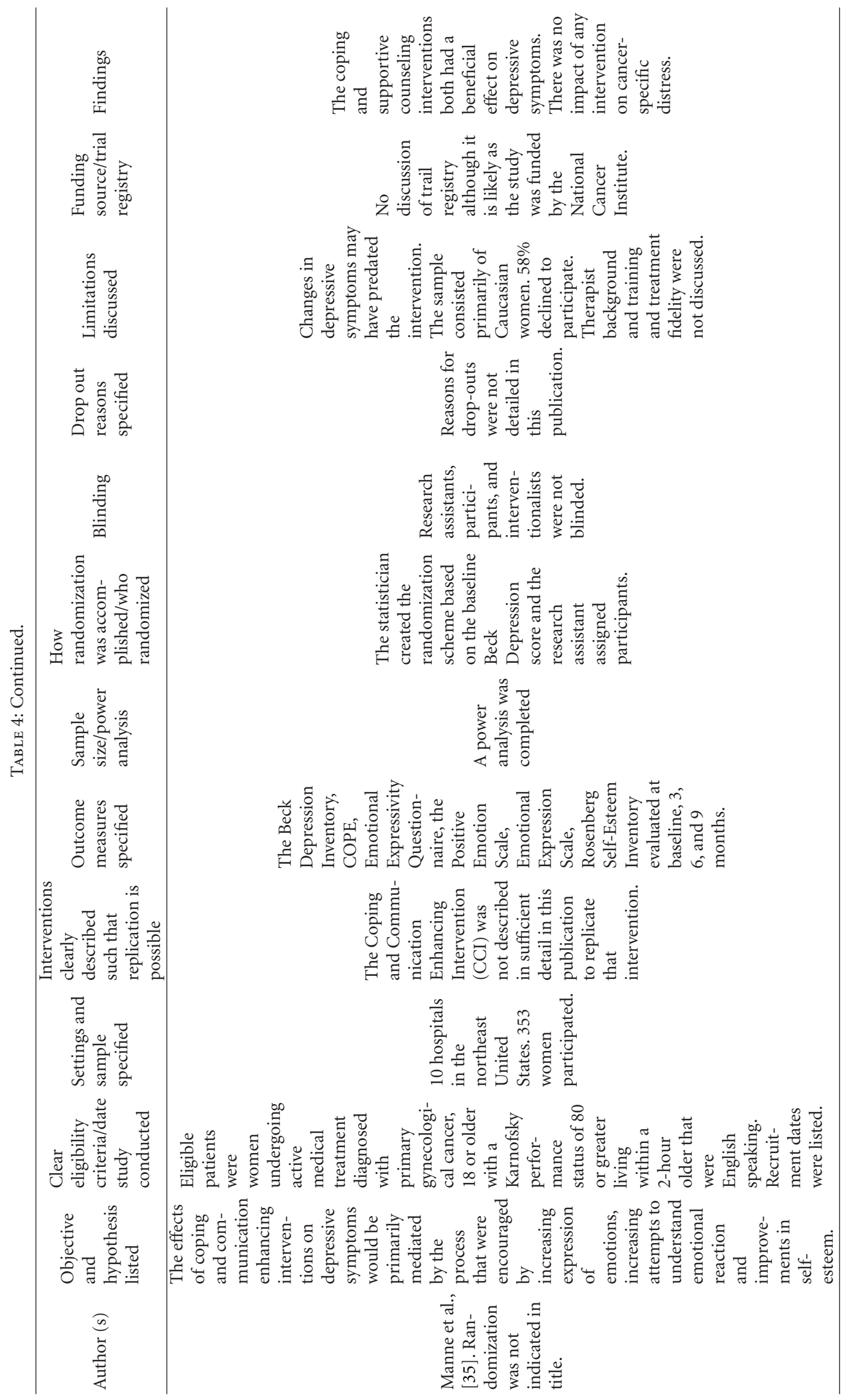




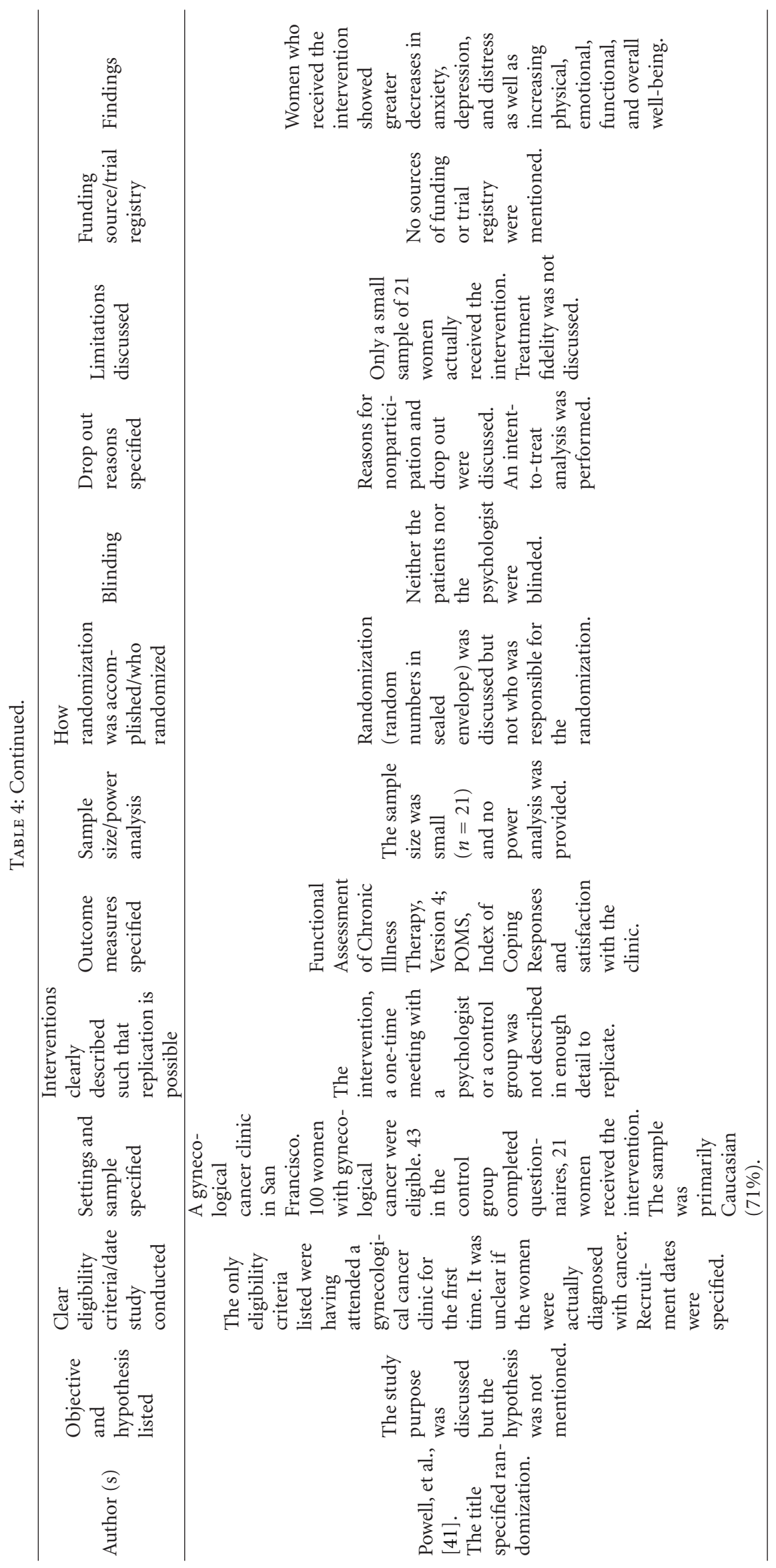




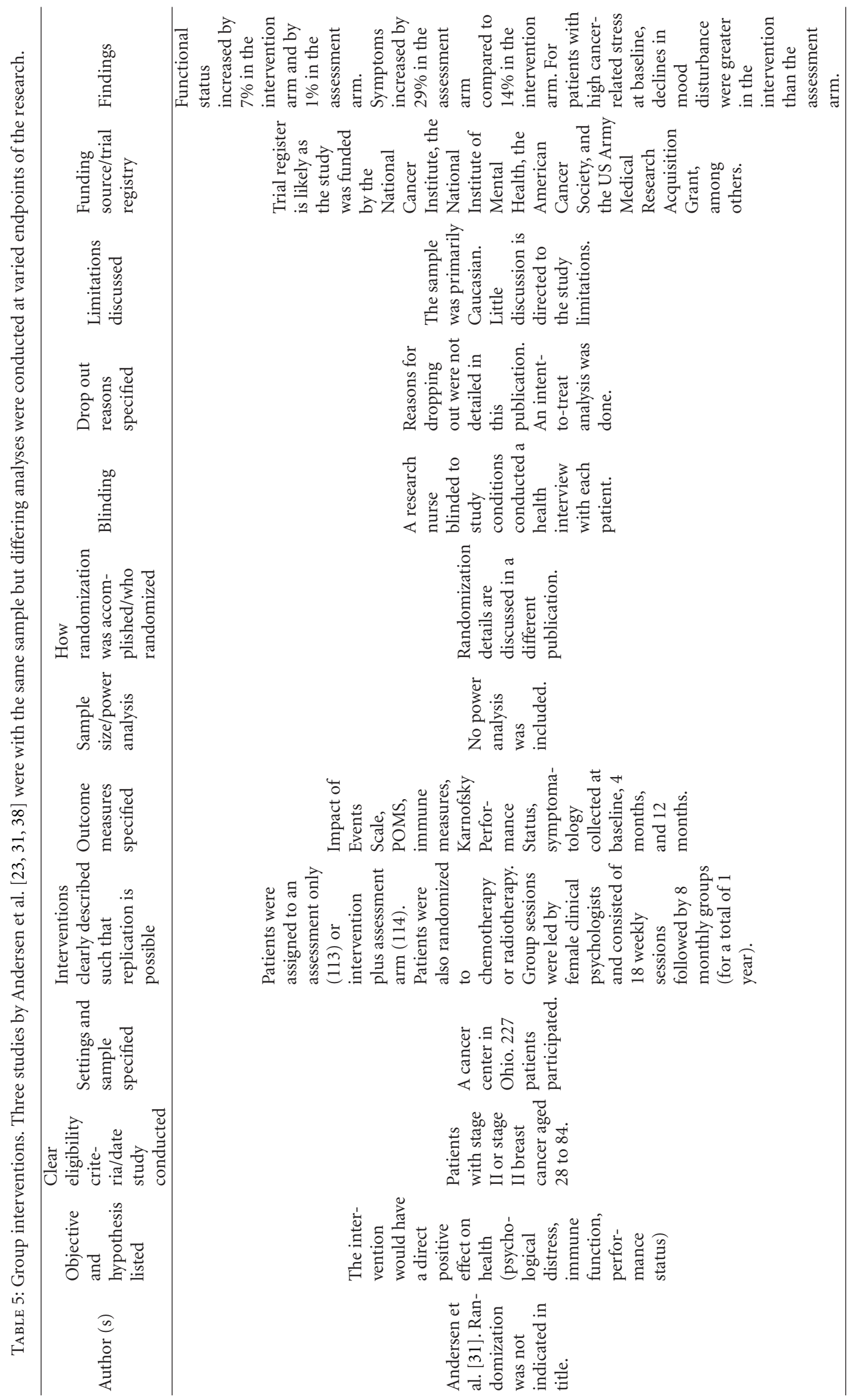




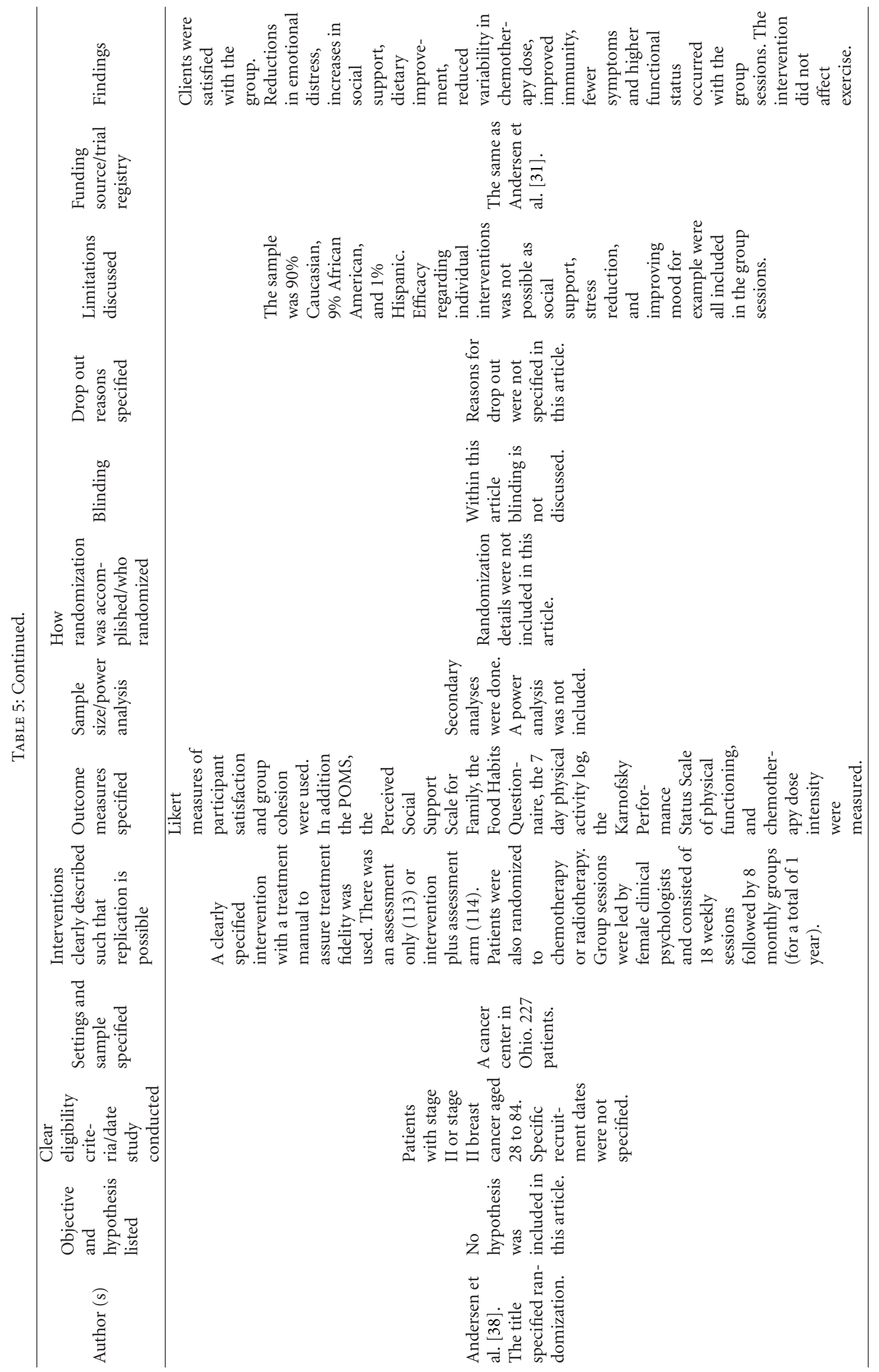




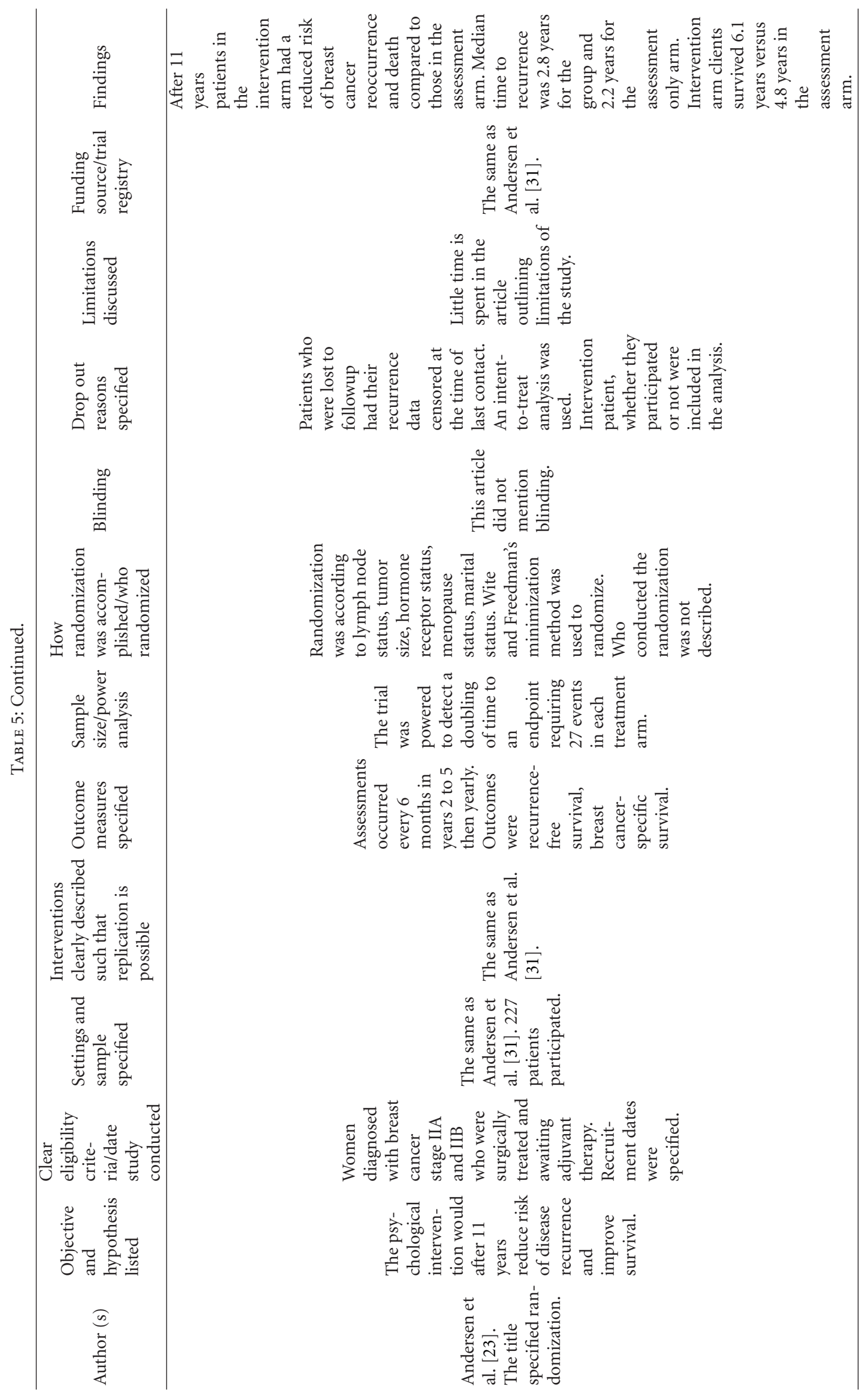




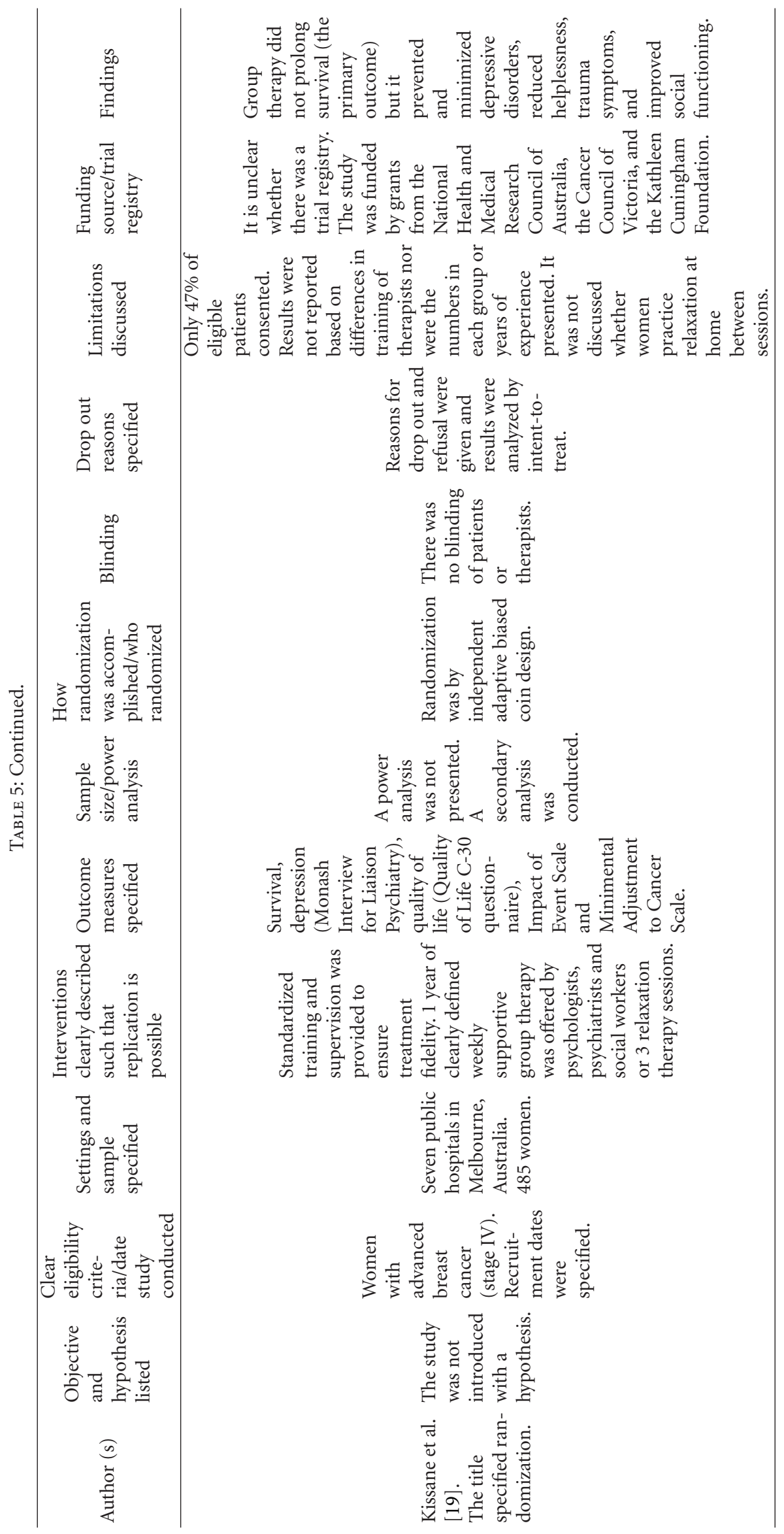




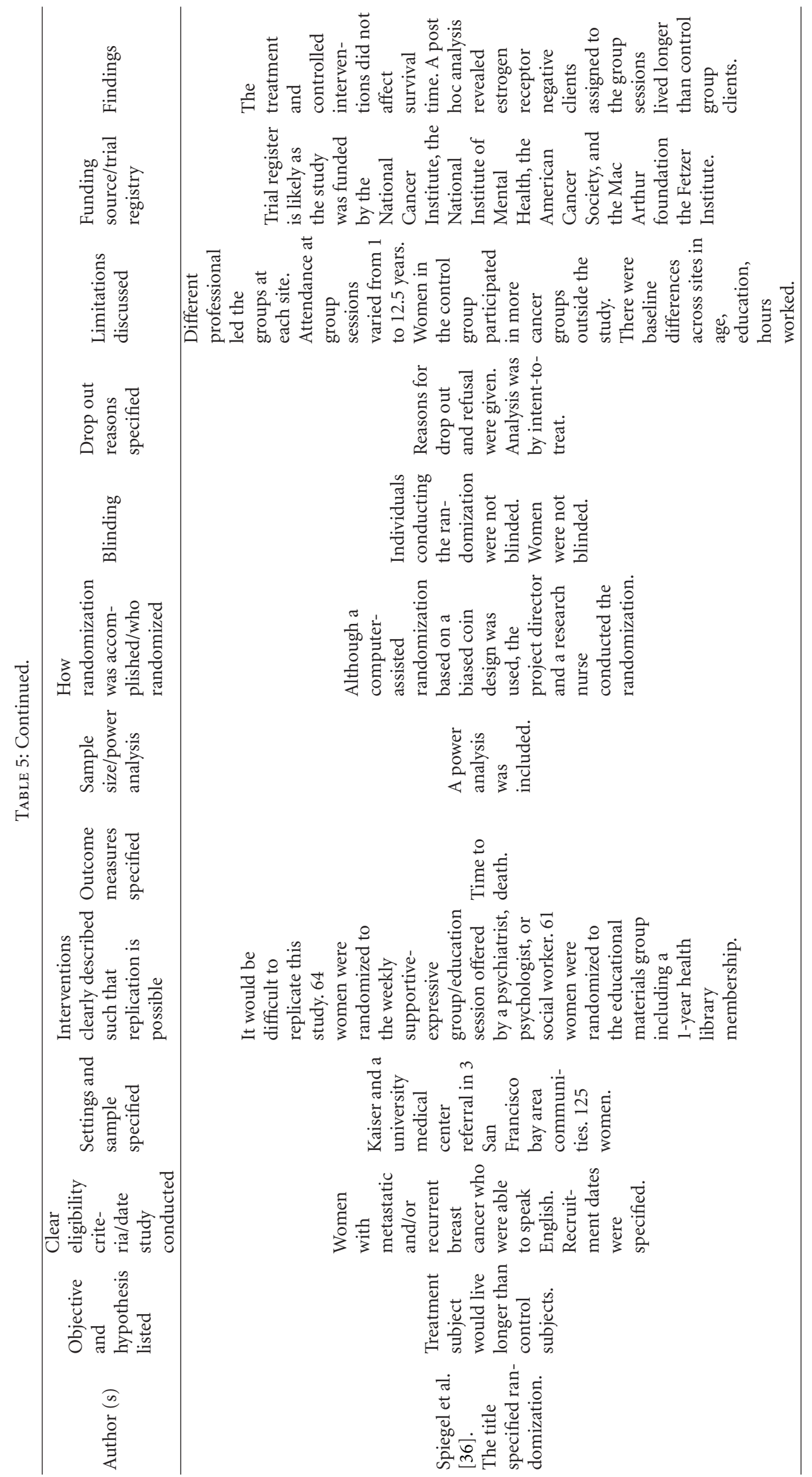




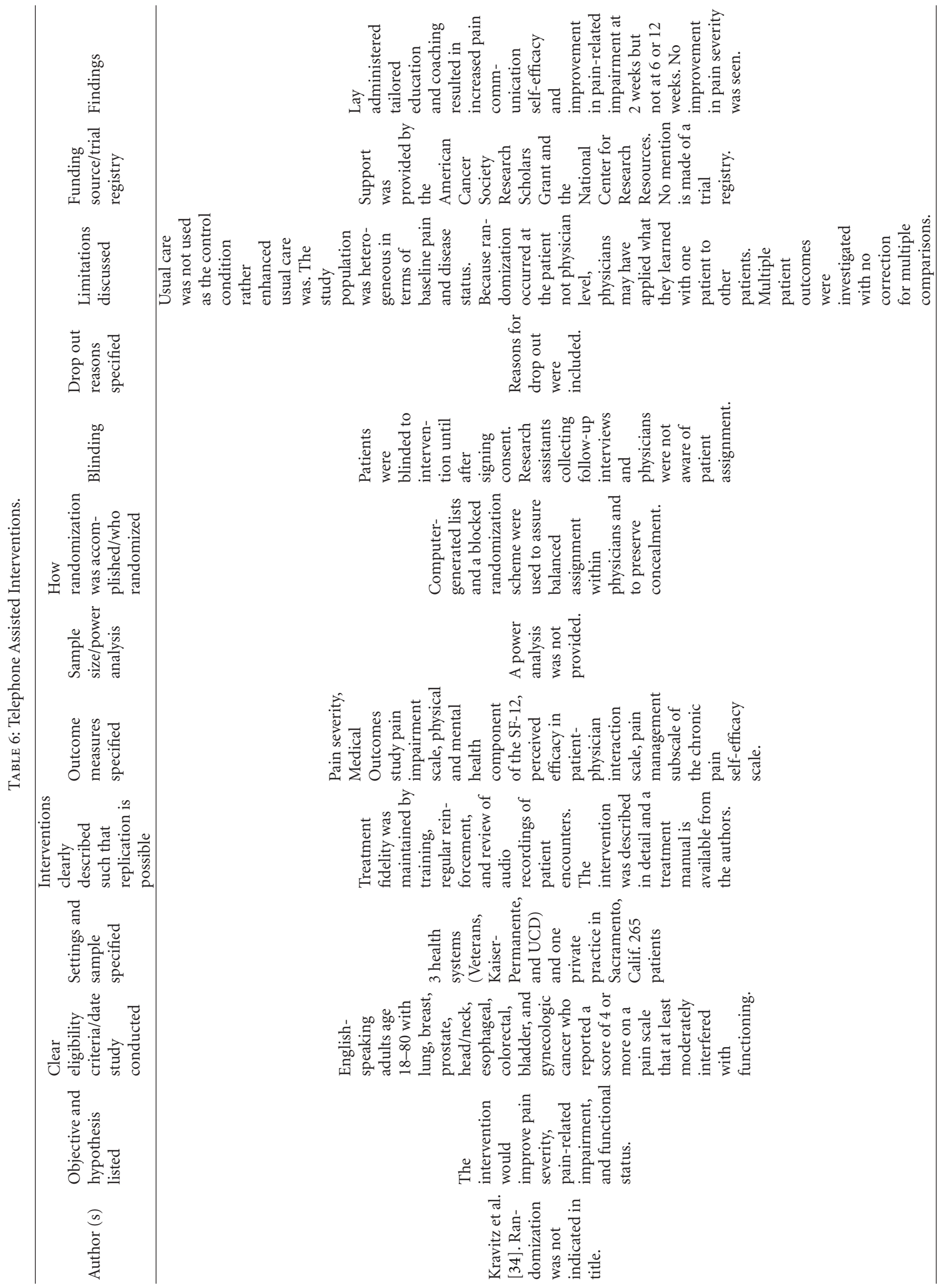




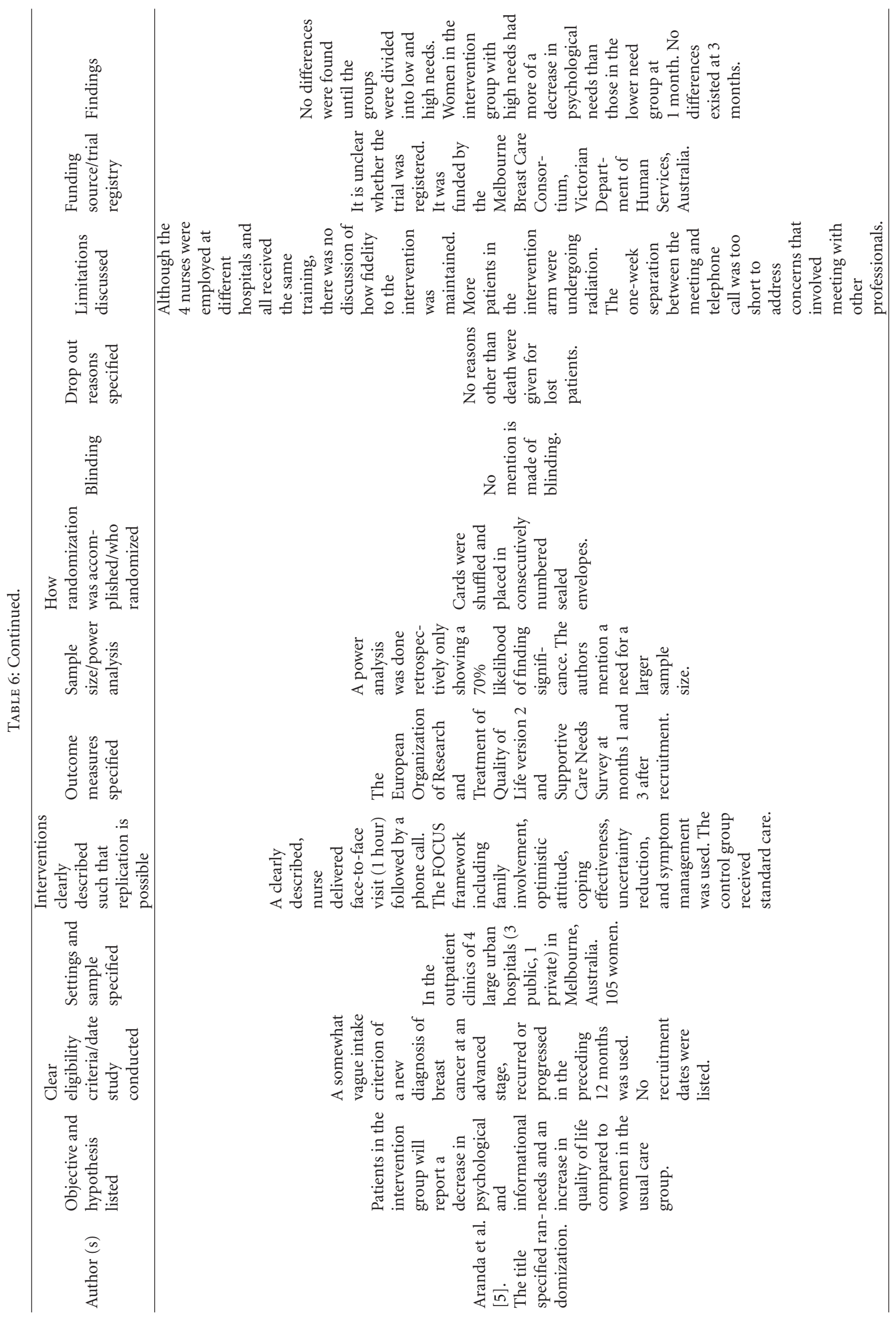




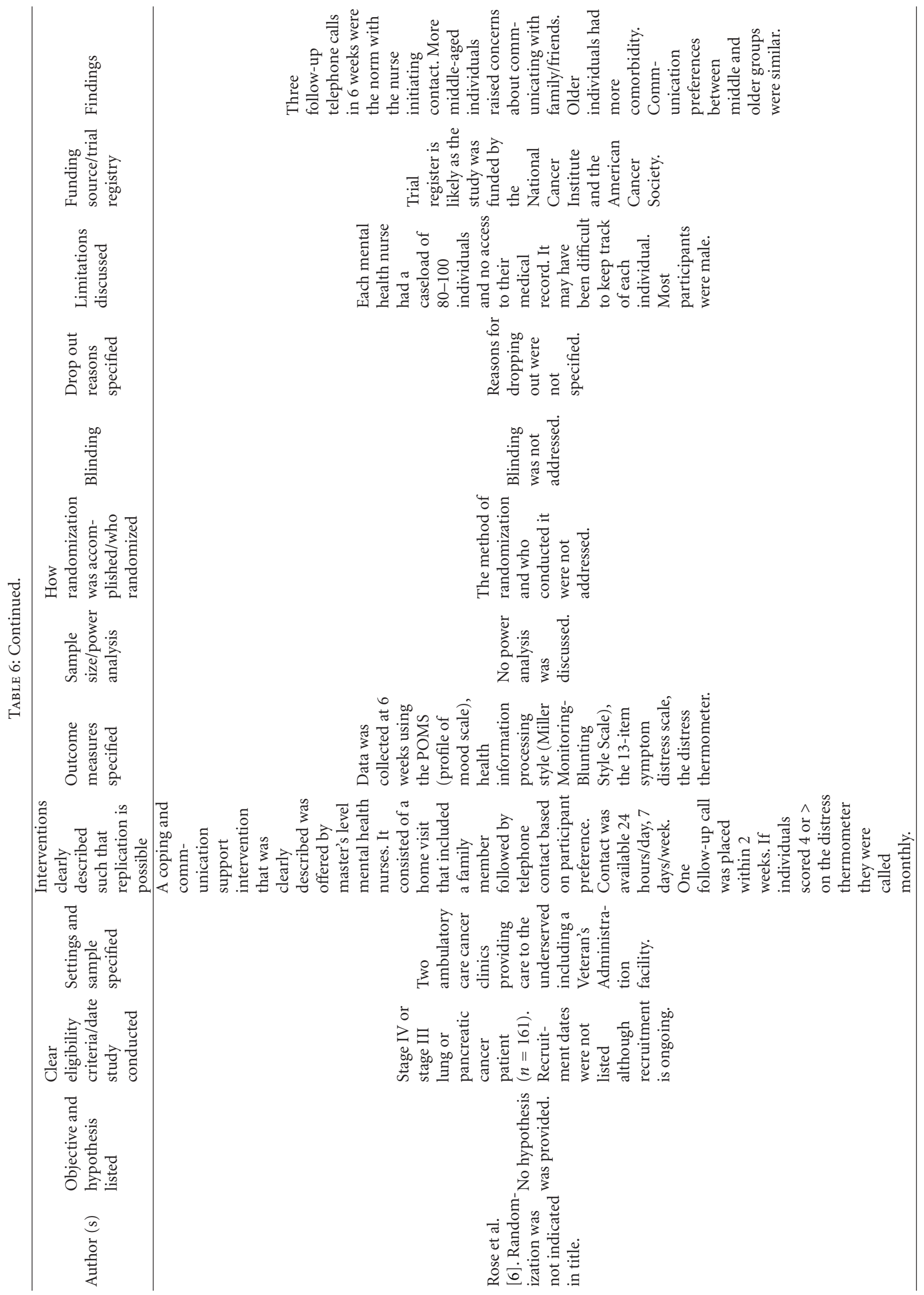




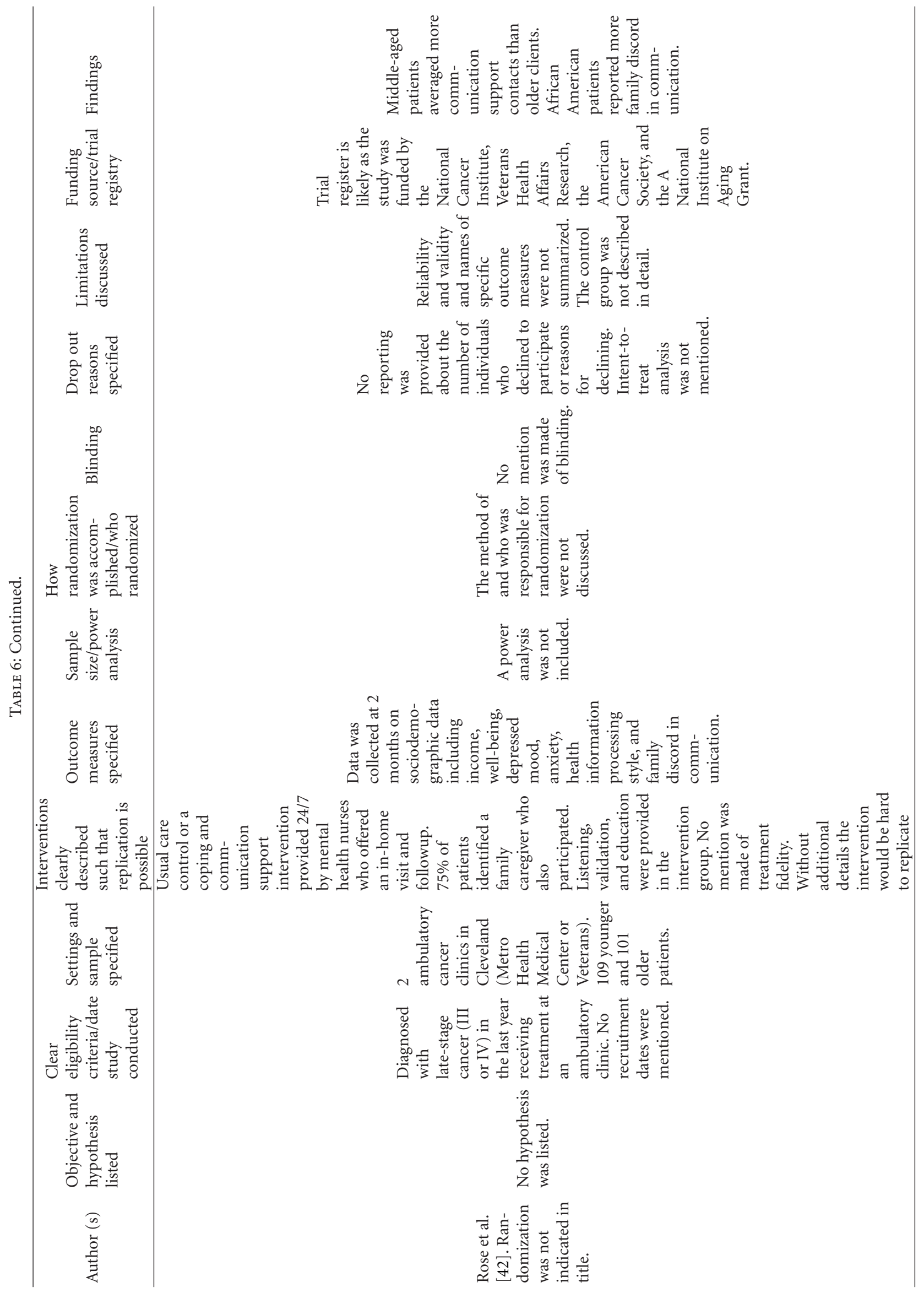




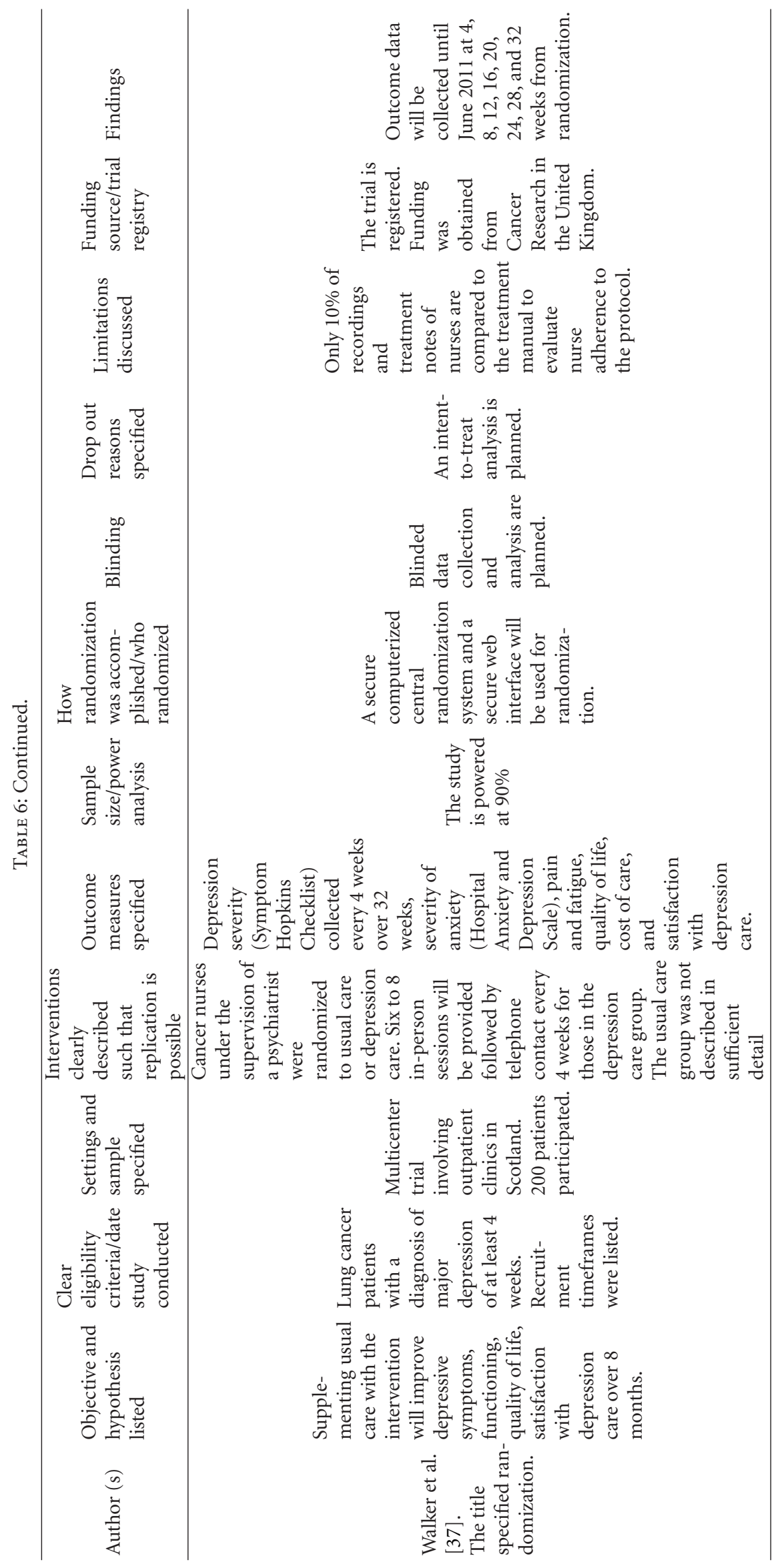




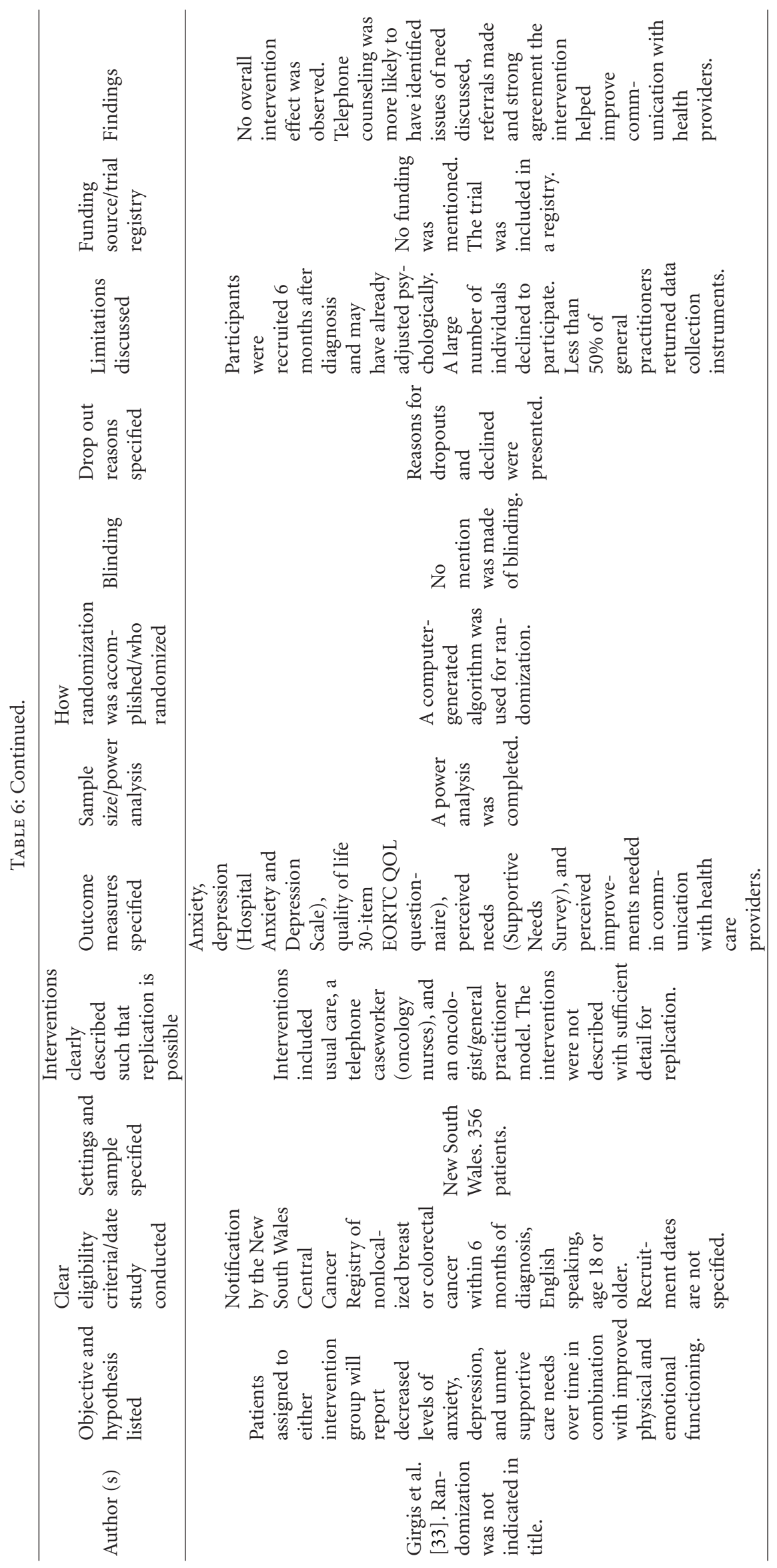


Walker et al. [37] randomized 200 individuals with lung cancer to a usual care or depression care intervention. Nurses offered 6 to 8 in-person sessions followed by monthly telephone contact. Results are pending as data collection is continuing until June of 2011.

Kravitz et al. [34] compared telephone intervention with care provided by a physician to find that short-term outcomes measured at 2 weeks improved although the longterm outcomes at 6 and 12 weeks were not improved. Girgis et al. [33] compared telephone caseworkers (oncology nurses) to a general practitioner model to find no overall intervention effect.

Additional research on the frequency, timing of in relation to diagnosis, and length of telephone support is needed to determine what level of intervention is needed. In addition, whether regional and generational differences exist in response to telephone support needs to be examined [20].

4.5. Overall Conclusions Regarding the Effectiveness of Psychosocial Interventions. Overall, 11 of the 19 studies included in this integrative review showed positive results $[7,23$, $25,26,31,32,34,35,38,40,41]$, and an additional 4 showed positive results $[5,19,33,36]$ based on a post hoc analysis. Consistent outcomes that were affected by more than one study in this integrative review included decreased depression $[19,26,35,41]$, decreased anxiety [7, $26,32,41]$, improved quality of life $[25,41]$, and enhanced functional status/well-being $[31,38]$. These positive results demonstrating the effectiveness of psychosocial interventions are consistent with positive results from previous metaanalyses $[9,12,16,17,45]$ and integrative reviews [11, 13]. In contrast, one previous integrative review reported that psychosocial interventions do not improve depression [15] while two integrative reviews reported psychosocial interventions do not prolong life $[14,18]$. This outcome of not increasing survival time is consistent with findings from this integrative review that showed survival time in two studies did not increase in the group receiving psychosocial interventions $[19,36]$ while one study reported a decreased risk of cancer reoccurrence in the group receiving psychosocial intervention [9]. Several authors of meta-analyses and integrative reviews have stressed the need for greater consistency within the research trajectory in this area in order to report conclusively that psychosocial interventions are effective $[15,18]$.

4.6. A Summary Regarding the Quality of Studies Reviewed. Variations among outcome measures, treatment conditions (cancer stage, treatment type, treatment location, and group/ individual approach), age, racial/ethnic background, and methodological quality made it difficult to draw definitive conclusions from this integrative review. What is clear is that both clinicians and researchers would benefit from future studies which build on previous findings, use comparable outcome measures, and adhere to standards of quality research such as those identified in the CONSORT guidelines [22]. It is important to identify what types and duration of intervention are effective for what population. As Jacobsen and Jim [17] commented "psychosocial care that is ineffective may be worse than no care at all" (p. 214). All of the 19 studies reviewed included detailed information about the analysis phase of their research. But as is evident in Table 2, not all of the studies reviewed included all of the quality measures that could be expected in a randomized, controlled clinical trial. Fewer studies (8 out of 19) included a power analysis, fewer studies addressed treatment fidelity (8 out of 19), fewer studies (9 out of 19) specified the method of randomization, fewer studies (5 out of 19) discussed blinding, fewer studies ( 9 out of 19) described reasons for dropping out of the study, and fewer studies (8 out of 19) used an intent-to-treat analysis.

Researchers need to include the quality measures listed in the CONSORT guidelines [22], incorporate cancer-specific outcomes measures, and explore whether certain interventions are more effective in select racial/ethnic or age groups. Additional research in needed to clarify if differences in time of diagnosis, cancer stage, the influence of age, or geographic area impact the effectiveness of psychosocial interventions.

It is useful to examine individual study quality by comparing a perfect score of 13 based on allocation of 1 point based on whether (1) a hypothesis was provided, (2) eligibility criteria are clear, (3) the intervention is described in sufficient detail for replication, (4) treatment fidelity is discussed, (5) a power analysis was provided, (6) the method of randomization was clear, (7) blinding was discussed, (8) reasons for dropouts were given, (9) an intent-to-treat analysis was used, (10) recruitment dates were specified, (11) study limitations were described, (12) funding sources were listed, and (13) a trial registry was mentioned. These 13 criteria included in the CONSORT guidelines [22] and summarized in Table 2 provide guidance when ranking study quality. Using these 13-point criteria Pitceathy et al. [26] and Walker et al. [37] received high scores of 11, followed by a score of 9 for Kravitz et al. [34], Spiegel et al. [36], and Kissane et al. [19] and by a score of 8 achieved by Andersen et al. [23] and Girgis et al. [33]. A midrange score of 7 is observed for Andersen et al. [31], Antoni et al. [32], Manne et al. [35], and Fukui et al. [40] while a score of 6 is seen for Antoni et al. [7] and Miller et al. [25]. Lower quality scores of 5 are observed for Aranda et al. [5], Andersen et al. [38], Boesen et al. [39], and Powell et al. [41]. The lowest quality scores of 3 for Rose et al. [42] and 4 for Rose et al. [6] are also evident. It is, however, important to remember that not all of the above-mentioned scores obtained from the CONSORT guidelines [22] are of strictly equivalent value for determining study quality.

Only eight studies $[19,26,32,34,36-38,40]$ did an adequate job of assuring treatment fidelity including (1) basing interventions on theory, (2) offering standardized training to those implementing the intervention, (3) monitoring to ensure interventions were consistently provided, (4) reinforcing training to avoid loss of skills or variation in approach, and (5) minimizing contact between treatment and control groups [46]. 
Too few studies have included mental health nurses or been designed by mental health nurses [6, 42]. Although it is a challenge given current economic realities, there is a need for mental health nurses to locate funding for randomized clinical trials and to focus on the effectiveness of mainstay interventions within mental health nursing practice, namely, therapeutic communication and supportive care in minimizing depressive symptoms and improving quality of life measures among individuals who have been diagnosed with cancer.

Eleven studies documented positive outcomes from psychological interventions $[7,23,25,26,31,32,34,35$, $38,40,41]$ and an additional 4 showed positive results $[5,19,33,36]$ based on ad hoc analysis. Data collection is continuing for 2 studies $[6,37]$. Additional research is needed because the literature continues to be full of methodological gaps and discrepancies. As Edwards et al. commented [18] it is difficult to integrate the results of randomized, controlled trials because of the variability of interventions, age groups, ethnic/racial backgrounds, cancer stages, outcome measures, and methodological quality. It is critical to increase the number of qualitative studies to determine what sort of intervention cancer patients of varied ages, ethnic/racial backgrounds, and cancer stages feel would meet their psychosocial needs. When those perspectives have been assessed researchers can design more effective randomized clinical trials that help close existing gaps in the literature.

\section{References}

[1] K. Ell, B. Quon, D. I. Quinn et al., "Improving treatment of depression among low-income patients with cancer: the design of the ADAPt-C study," General Hospital Psychiatry, vol. 29, no. 3, pp. 223-231, 2007.

[2] S. Kaasa, U. Malt, S. Hagen, E. Wist, T. Moum, and A. Kvikstad, "Psychological distress in cancer patients with advanced disease," Radiotherapy and Oncology, vol. 27, no. 3, pp. 193197, 1993.

[3] J. Zabora, K. Brintzenhofeszoc, B. Curbow, C. Hooker, and S. Piantadosi, "The prevalence of psychological distress by cancer site," Psycho-Oncology, vol. 10, no. 1, pp. 19-28, 2001.

[4] J. Turner, S. Zapart, K. Pedersen, N. Rankin, K. Luxford, and J. Fletcher, "Clinical practice guidelines for the psychosocial care of adults with cancer," Psycho-Oncology, vol. 14, no. 3, pp. 159-173, 2005.

[5] S. Aranda, P. Schofield, L. Weih, D. Milne, P. Yates, and R. Faulkner, "Meeting the support and information needs of women with advanced breast cancer: a randomised controlled trial," British Journal of Cancer, vol. 95, no. 6, pp. 667-673, 2006.

[6] J. H. Rose, R. Radziewicz, K. F. Bowman, and E. E. O’Toole, "A coping and communication support intervention tailored to older patients diagnosed with late-stage cancer," Clinical Interventions in Aging, vol. 3, no. 1, pp. 77-95, 2008.

[7] M. H. Antoni, S. R. Wimberly, S. C. Lechner et al., "Reduction of cancer-specific thought intrusions and anxiety symptoms with a stress management intervention among women undergoing treatment for breast cancer," American Journal of Psychiatry, vol. 163, no. 10, pp. 1791-1797, 2006.

[8] H. Seow, C. F. Snyder, L. R. Shugarman et al., "Developing quality indicators for cancer end-of-life care: proceedings from a national symposium," Cancer, vol. 115, no. 17, pp. 3820-3829, 2009.

[9] T. J. Meyer and M. M. Mark, "Effects of psychosocial interventions with adult cancer patients: a meta-analysis of randomized experiments," Health Psychology, vol. 14, no. 2, pp. 101-108, 1995.

[10] S. A. Newell, R. W. Sanson-Fisher, and N. J. Savolainen, "Systematic review of psychological therapies for cancer patients: Overview and recommendations for future research," Journal of the National Cancer Institute, vol. 94, no. 8, pp. 558-584, 2002.

[11] A. M. Barsevick, C. Sweeney, E. Haney, and E. Chung, "A systematic qualitative analysis of psychoeducational interventions for depression in patients with cancer," Oncology nursing forum, vol. 29, no. 1, pp. 73-85, 2002.

[12] B. Rehse and R. Pukrop, "Effects of psychosocial interventions on quality of life in adult cancer patients: Meta analysis of 37 published controlled outcome studies," Patient Education and Counseling, vol. 50, no. 2, pp. 179-186, 2003.

[13] R. J. Uitterhoeve, M. Vernooy, M. Litjens et al., "Psychosocial interventions for patients with advanced cancer: a systematic review of the literature," British Journal of Cancer, vol. 91, no. 6, pp. 1050-1062, 2004.

[14] E. Chow, M. N. Tsao, and T. Harth, "Does psychosocial intervention improve survival in cancer? A meta-analysis," Palliative Medicine, vol. 18, no. 1, pp. 25-31, 2004.

[15] S. Williams and J. Dale, "The effectiveness of treatment for depression/depressive symptoms in adults with cancer: a systematic review," British Journal of Cancer, vol. 94, no. 3, pp. 372-390, 2006.

[16] R. L. Osborn, A. C. Demoncada, and M. Feuerstein, "Psychosocial interventions for depression, anxiety, and quality of life in cancer survivors: meta-analyses," International Journal of Psychiatry in Medicine, vol. 36, no. 1, pp. 13-34, 2006.

[17] P. B. Jacobsen and H. S. Jim, "Psychosocial interventions for anxiety and depression in adult cancer patients: achievements and challenges," CA Cancer Journal for Clinicians, vol. 58, no. 4, pp. 214-230, 2008.

[18] A. G. Edwards, N. Hulbert-Williams, and R. D. Neal, "Psychological interventions for women with metastatic breast cancer," The Cochrane Library, no. 3, 2008.

[19] D. W. Kissane, B. Grabsch, D. M. Clarke et al., "Supportiveexpressive group therapy for women with metastatic breast cancer: survival and psychosocial outcome from a randomized controlled trial," Psycho-Oncology, vol. 16, no. 4, pp. 277-286, 2007.

[20] M. C. Wilmoth, L. Tulman, E. A. Coleman, C. B. Stewart, and N. Samarel, "Women's perceptions of the effectiveness of telephone support and education on their adjustment to breast cancer," Oncology Nursing Forum, vol. 33, no. 1, pp. 138-144, 2006.

[21] S. Hall, H. Chochinov, R. Harding, S. Murray, A. Richardson, and I. J. Higginson, "A Phase II randomised controlled trial assessing the feasibility, acceptability and potential effectiveness of Dignity Therapy for older people in care homes: study protocol," BMC Geriatrics, vol. 9, no. 1, article 9, 2009, http://www.biomedcentral.com/1471-2318/9/9.

[22] K. F. Schulz, D. G. Altman, and D. Moher, "CONSORT 2010 statement: updated guidelines for reporting parallel group randomized trials," Annals of Internal Medicine, vol. 152, no. 11, pp. 726-732, 2010.

[23] B. L. Andersen, H. C. Yang, W. B. Farrar et al., "Psychologic intervention improves survival for breast cancer patients: a 
randomized clinical trial," Cancer, vol. 113, no. 12, pp. 3450 $3458,2008$.

[24] L. J. Edgar, J. Remmer, Z. Rosberger, and B. Rapkin, "Evaluating a volunteer cancer support service," Journal of Psychosocial Oncology, vol. 21, no. 1, pp. 53-72, 2003.

[25] J. J. Miller, M. H. Frost, T. A. Rummans et al., "Role of a medical social worker in improving quality of life for patients with advanced cancer with a structured multidisciplinary intervention," Journal of Psychosocial Oncology, vol. 25, no. 4, pp. 105-119, 2007.

[26] C. Pitceathly, P. Maguire, I. Fletcher, M. Parle, B. Tomenson, and F. Creed, "Can a brief psychological intervention prevent anxiety or depressive disorders in cancer patients? A randomised controlled trial," Annals of Oncology, vol. 20, no. 5, pp. 928-934, 2008.

[27] L. D. Butler, C. Koopman, E. Neri et al., "Effects of SupportiveExpressive Group Therapy on Pain in Women With Metastatic Breast Cancer," Health Psychology, vol. 28, no. 5, pp. 579-587, 2009.

[28] T. A. Rummans, M. M. Clark, J. A. Sloan et al., "Impacting quality of life for patients with advanced cancer with a structured multidisciplinary intervention: a randomized controlled trial," Journal of Clinical Oncology, vol. 24, no. 4, pp. 635-642, 2006.

[29] S. Manne, J. S. Ostroff, and G. Winkel, "Social-cognitive processes as moderators of a couple-focused group intervention for women with early stage breast cancer," Health Psychology, vol. 26, no. 6, pp. 735-744, 2007.

[30] K. Ell, B. Xie, B. Quon, D. I. Quinn, M. Dwight-Johnson, and P. J. Lee, "Randomized controlled trial of collaborative care management of depression among low-income patients with cancer," Journal of Clinical Oncology, vol. 26, no. 27, pp. 44884496, 2008.

[31] B. L. Andersen, W. B. Farrar, D. Golden-Kreutz et al., "Distress reduction from a psychological intervention contributes to improved health for cancer patients," Brain, Behavior, and Immunity, vol. 21, no. 7, pp. 953-961, 2007.

[32] M. H. Antoni, S. Lechner, A. Diaz et al., "Cognitive behavioral stress management effects on psychosocial and physiological adaptation in women undergoing treatment for breast cancer," Brain, Behavior, and Immunity, vol. 23, no. 5, pp. 580-591, 2008.

[33] A. Girgis, S. Breen, F. Stacey, and C. Lecathelinais, "Impact of two supportive care interventions on anxiety, depression, quality of life, and unmet needs in patients with nonlocalized breast and colorectal cancers," Journal of Clinical Oncology, vol. 27, no. 36, pp. 6180-6190, 2009.

[34] R. L. Kravitz, D. J. Tancredi, T. Grennan et al., "Cancer health empowerment for living without pain (Ca-HELP): effects of a tailored education and coaching intervention on pain and impairment," Pain, vol. 152, no. 7, pp. 1572-1582, 2011.

[35] S. L. Manne, G. Winkel, S. Rubin et al., "Mediators of a Coping and Communication-Enhancing Intervention and a Supportive Counseling Intervention Among Women Diagnosed With Gynecological Cancers," Journal of Consulting and Clinical Psychology, vol. 76, no. 6, pp. 1034-1045, 2008.

[36] D. Spiegel, L. D. Butler, J. Giese-Davis et al., "Effects of supportive-expressive group therapy on survival of patients with metastatic breast cancer: a randomized prospective trial," Cancer, vol. 110, no. 5, pp. 1130-1138, 2007.

[37] J. Walker, J. Cassidy, and M. Sharpe, "The third symptom management research trial in oncology (SMaRT Oncology-3): a randomised trial to determine the efficacy of adding a complex intervention for major depressive disorder (Depression Care for People with Lung Cancer) to usual care, compared to usual care alone in patients with lung cancer," Trials, vol. 10, article 1745, p. 92, 2009, http://www.trialsjournal.com/content/10/1/92.

[38] B. L. Andersen, R. A. Shelby, and D. M. Golden-Kreutz, "RCT of a psychological intervention for patients with cancer: $i$. mechanisms of change," Journal of Consulting and Clinical Psychology, vol. 75, no. 6, pp. 927-938, 2007.

[39] E. Boesen, S. Boesen, S. Christensen, and C. Johansen, "Comparison of participants and non-participants in a randomized psychosocial intervention study among patients with malignant melanoma," Psychosomatics, vol. 48, no. 6, pp. 510-516, 2007.

[40] S. Fukui, K. Ogawa, M. Ohtsuka, and N. Fuku, "A randomized study assessing the efficacy of communication skill training on patients' psychologic distress and coping nurses' communication with patients just after being diagnosed with cancer," Cancer, vol. 113, no. 6, pp. 1462-1470, 2008.

[41] C. B. Powell, A. Kneier, L. M. Chen, M. Rubin, C. Kronewetter, and E. Levine, "A randomized study of the effectiveness of a brief psychosocial intervention for women attending a gynecologic cancer clinic," Gynecologic Oncology, vol. 111, no. 1, pp. 137-143, 2008.

[42] J. H. Rose, K. F. Bowman, R. M. Radziewicz, S. A. Lewis, and E. E. O'Toole, "Predictors of engagement in a coping and communication support intervention for older patients with advanced cancer," Journal of the American Geriatrics Society, vol. 57, no. 2, pp. S296-S299, 2009.

[43] F. I. Fawzy and N. W. Fawzy, "A structured psychoeducational intervention for cancer patients," General Hospital Psychiatry, vol. 16, no. 3, pp. 149-192, 1994.

[44] E. H. Boesen, L. Ross, and K. Frederiksen, "A psycho-educational intervention for patients with cutaneous malignant melanoma: a replication study of Fawzy et al," Clinical Oncology, vol. 23, pp. 1270-1277, 2005.

[45] A. Zabalegui, S. Sanchez, P. D. Sanchez, and C. Juando, "Integrative literature reviews and meta-analyses: nursing and cancer support groups," Journal of Advanced Nursing, vol. 51, no. 4, pp. 369-381, 2005.

[46] R. M. Radziewicz, J. H. Rose, K. F. Bowman, R. A. Berila, E. E. O’Toole, and B. Given, "Establishing treatment fidelity in a coping and communication support telephone intervention for aging patients with advanced cancer and their family caregivers," Cancer Nursing, vol. 32, no. 3, pp. 193-202, 2009. 


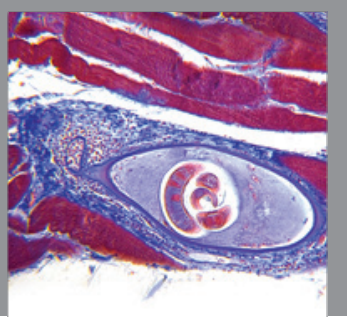

Gastroenterology

Research and Practice
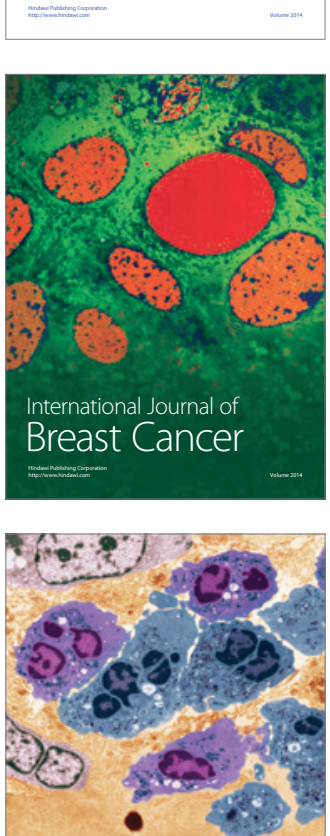

International Journal of Inflammation
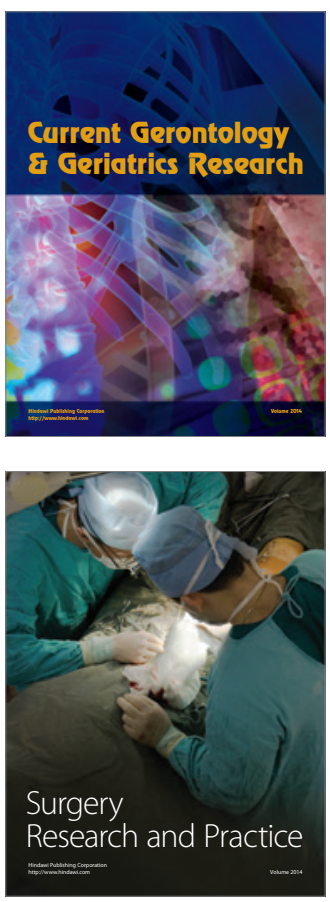
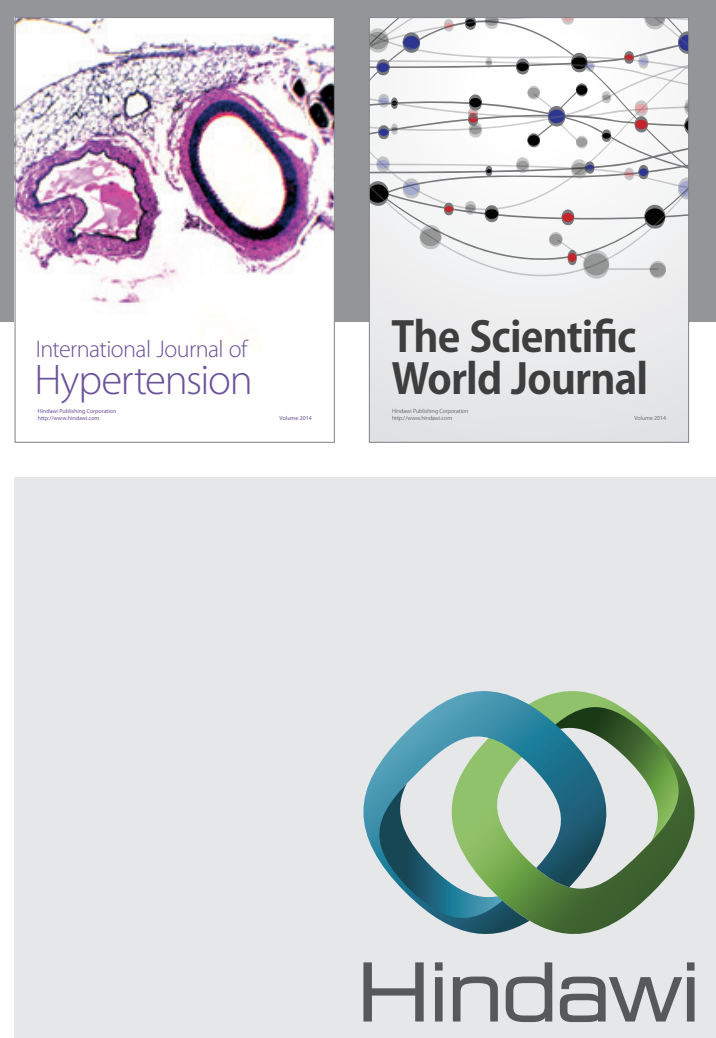

Submit your manuscripts at

http://www.hindawi.com
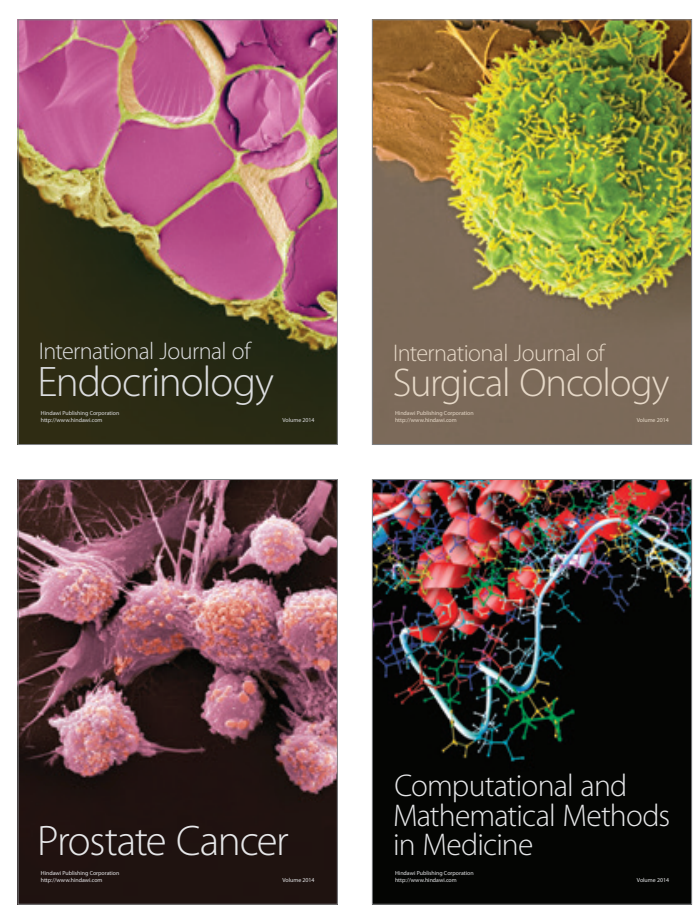
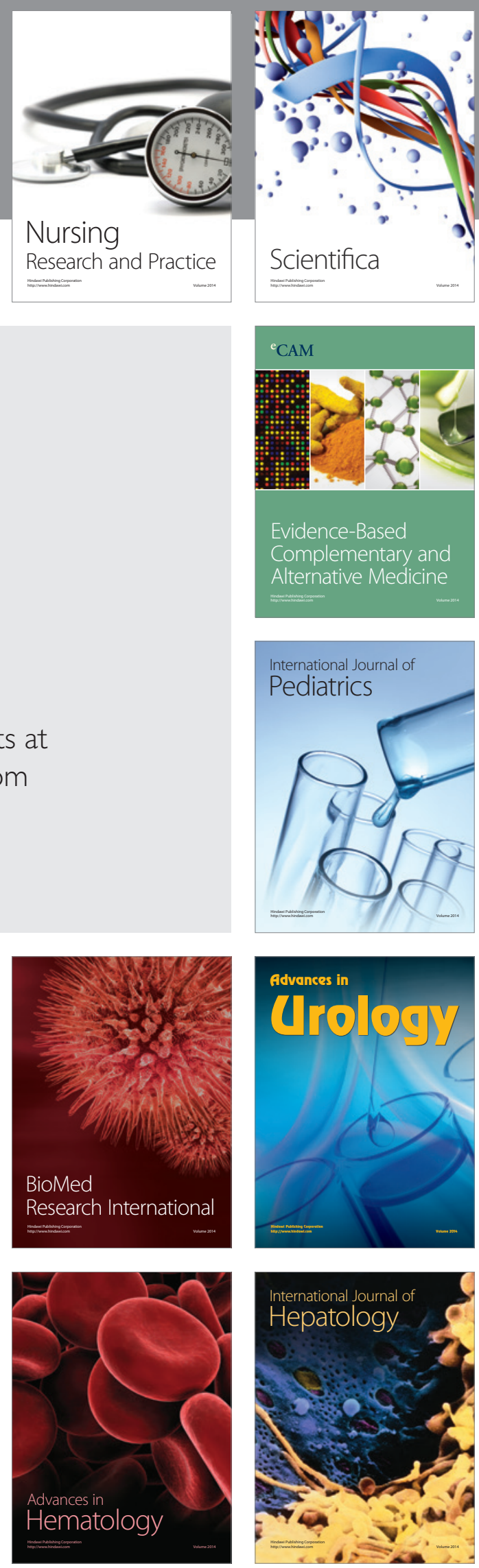